\title{
Recent advances in flexible supercapacitors based on carbon nanotubes and graphene
}

\author{
Kang Li and Jintao Zhang*
}

\begin{abstract}
Owing to the rapidly growing market for flexible electronics, there is an urgent demand to develop flexible energy storage devices. Flexible supercapacitors have received much attention due to their good flexibility, fast charge/discharge rate and long lifecycle times. Carbon nanotubes (CNTs) and graphene have good mechanical properties, which make them suitable for flexible supercapacitors. Based on different nanostructures of CNTs and graphene, we summarized the recent progress in CNTs- and graphene-based flexible supercapacitors with a brief description of the basic principles for evaluating their performance. Special emphasis was given to fabrication methods, capacitive performance and electrode configurations of different flexible supercapacitors. Furthermore, the remaining challenges and future research directions for CNTs- and graphene-based flexible supercapacitors have also been discussed.
\end{abstract}

Keywords: flexible supercapacitor, carbon nanotubes, graphene, nanostructure

\section{INTRODUCTION}

Due to the limited reserves of fossil fuels, the exploitation of clean and sustainable energy in form of solar, wind and geothermal is critical to solving the energy crisis and ameliorating environmental problems. To more easily and affordably use the above-mentioned energy sources, energy storage devices must be dramatically improved [15]. Rechargeable batteries and supercapacitors are the two most commonly used devices for energy storage, and have attracted persistent research attention over the last few decades [6-9].

With the emergence of flexible and wearable electronics, there is increasing demand for energy storage devices with similar properties [10-14]. However, conventional batteries and supercapacitors cannot meet the requirements for powering flexible electronics due to their heavy and rigid outer shells. The rigid structures prevent the bending, twisting, and stretching of conventional batteries, which are the basic features for flexible electronics. In addition, traditional electrode fabrication processes involve a slurry-making process, during which insulative binders and conductive additives are used. The binders and conductive additives inhibits the electrochemical performance of batteries and supercapacitors because they contribute little to the overall performance, while constituting $20-30 \mathrm{wt} . \%$ of the slurry [15-18]. In addition, the liquid electrolyte of traditional energy storage devices will leak when the battery is deformed, which poses serious safety concerns. Hence, it is highly desirable to develop novel flexible energy storage systems where all components are suitable for flexible electronics.

Supercapacitors, sometimes called ultracapacitors or electrocapacitors, are energy devices which can provide ultralong cycle life and fast charge/discharge rates. Typically, supercapacitors are divided into electrical doublelayer capacitors (EDLCs) and pseudocapacitor according to their energy storage mechanism. In EDLCs, energy is stored in their electrode/electrolyte interface by ion adsorption, whereas pseudocapacitors store energy by reversible surface or near-surface faradic reactions. Therefore, the specific capacitance of a pseudocapacitor is often larger than that of an EDLC. However, pseudocapacitors usually suffer from poor cycle stability and low rate performance due to the nature of their electrode materials (e.g., metal oxides). In comparison with batteries, both types of supercapacitors generally offer higher power densities, but lower energy densities. With the rapid development of flexible electronics, various elec-

Key Laboratory for Colloid and Interface Chemistry, Ministry of Education, School of Chemistry and Chemical Engineering, Shandong University, Jinan 250100, China

* Corresponding author (email: jtzhang@sdu.edu.cn) 
trode materials including carbon materials [7,19-21], metal oxides/hydroxides [22-24], conductive polymers [25-27] and their associated composites have been developed to fabricate flexible supercapacitors.

Carbon materials with flexible structural features including one-dimensional (1D) carbon nanotubes (CNTs) and two-dimensional (2D) graphene have been applied in many fields such as catalysis, batteries, and fuel cells. More importantly, carbon materials are suitable for constructing flexible supercapacitors due to their large surface area, high electrical conductivity, as well as good chemical and mechanical stability [28-35]. Several excellent reviews summarized the development of flexible electrodes for energy storage devices $[29,31,36]$. However, the development of flexible supercapacitors has been seen as significant advances in the past few years. Therefore, it is urgent to update the wider community on the recent achievements for configuring flexible supercapacitors based on CNTs and graphene. Herein, we summarize the recent progress in the development of flexible supercapacitors with special focus on the use of CNT- and graphene-based materials as flexible electrodes. First, we will briefly introduce the basic principles of performance evaluation of flexible supercapacitors. Then, the performance of flexible electrodes with various structures, such as fiber, film, and foam will be summarized with a focus on recent achievements in the fabrication of flexible supercapacitors based on CNTs and graphene. Emphasis is placed on the electrode preparation methods, electrochemical capacitive performance and flexibility tests. Finally, the remaining challenges and some perspective regarding future research directions in the field will be discussed.

\section{EVALUATION OF FLEXIBLE SUPERCAPACITOR PERFORMANCE}

Specific capacitance, energy density and power density are the three major metrics typically used to evaluate the electrochemical performances of a supercapacitor. A three-electrode system with working, counter, and reference electrode is often employed to study the fundamental electrochemical properties of an active material. However, when it comes to evaluating the integral cell performance, a two-electrode system is often more accurate because it resembles more closely the setup of a real supercapacitor. A two-electrode supercapacitor includes an electrolyte, a separator and two electrodes, as illustrated in Fig. 1. Each electrode/electrolyte interface can be regarded as a capacitor and a resistor, thus, the whole cell is considered as two capacitors in series. The

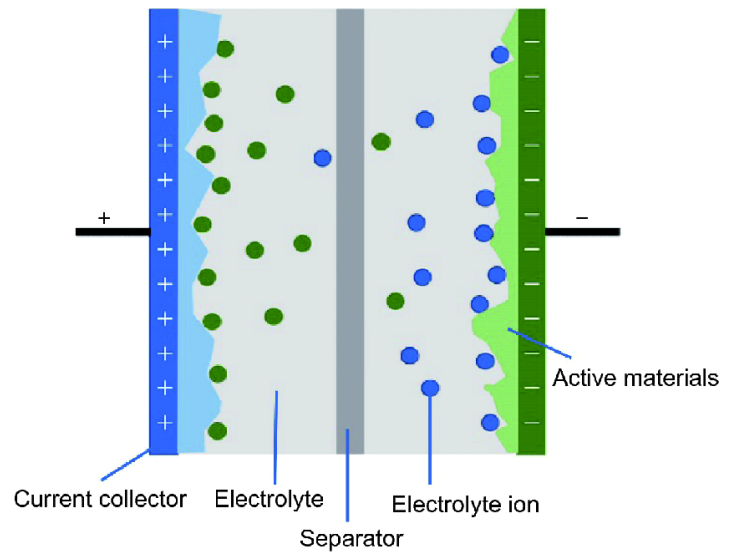

Figure 1 Schematic illustration of a two-electrode supercapacitor.

total capacitance of the cell $\left(C_{\text {cell }}\right)$ can be calculated according to Equation 1, where $C_{1}$ and $C_{2}$ are the capacitance of the two electrodes.

$$
\frac{1}{C_{\text {cell }}}=\frac{1}{C_{1}}+\frac{1}{C_{2}}
$$

Cyclic voltammetry (CV) and galvanostatic charge/ discharge (GCD) curves are basic methods to measure the electrochemical performance of a supercapacitor. Based on the CV curves, $C_{\text {cell }}$ can be calculated according to Equation 2:

$$
C_{\text {cell }}=\frac{Q}{2 V}=\frac{1}{2 V v} \int_{V_{-}}^{V_{+}} i(V) \mathrm{d} V,
$$

in which $Q$ is the total charge obtained by the integration of the positive and negative sweeps in a CV curve, $v$ is the scan rate, and $\left(V=V_{+}-V_{-}\right)$represents the potential window used during the scan process. Alternatively, $C_{\text {cell }}$ also can be determined by the GCD. Using this method, the capacitance of the cell can be calculated from the constant discharge curves according to Equation 3:

$$
C_{\text {cell }}=\frac{i}{\mathrm{~d} V / \mathrm{d} t},
$$

in which $i$ is the discharge current, $t$ is the discharge time and $V$ is the working potential without the IR drop. In addition, $\mathrm{d} V / \mathrm{d} t$ can be obtained from the slope of the discharge curve. To compare the capacitive performance of different materials or devices, specific capacitance $\left(C_{\mathrm{s}}\right)$, i.e., the capacitance per unit mass, length, area or volume, is widely used, and can be calculated according to Equation 4:

$$
C_{\mathrm{s}}=\frac{C_{\text {single }}}{U C_{i}}=2 \frac{C_{\text {cell }}}{U C_{i}},
$$

in which $C_{\text {single }}$ is the capacitance of a single electrode, for symmetric supercapacitors, $C_{\text {single }}=C_{1}=C_{2}=2 C_{\text {cell }} \cdot U C_{\mathrm{i}}$ is a unit conversion parameter depending on the specific 
capacitance required. For instance, if gravimetric specific capacitance $\left(\mathrm{F} \mathrm{g}^{-1}\right)$ is used, $U C_{\mathrm{i}}$ should be the mass $(\mathrm{g})$ whereas for volumetric specific capacitance $\left(\mathrm{F} \mathrm{cm}^{-3}\right), U C_{\mathrm{i}}$ should be the volume $\left(\mathrm{cm}^{3}\right)$. It should be noted that the reported value may depend on the active materials or the whole device, leading to significantly different performance.

The energy density $\left(E_{\mathrm{i}}\right)$ and power density $\left(P_{\mathrm{i}}\right)$ are two important parameters commonly used to evaluate the performance of a supercapacitor device. $E_{\mathrm{i}}$ reflects the capacity to release energy while $P_{\mathrm{i}}$ indicates how fast the energy can be delivered. $E_{\mathrm{i}}$ and $P_{\mathrm{i}}$ can be calculated according to Equations 5, 6, respectively:

$$
\begin{gathered}
E_{\mathrm{i}}=\frac{1}{2} \frac{C_{\text {cell }} V^{2}}{U C_{\mathrm{i}}}, \\
P_{\mathrm{i}}=\frac{1}{4} \frac{V^{2}}{U C_{\mathrm{i}} R_{\mathrm{ESR}}},
\end{gathered}
$$

in which $V$ is the cell operating voltage and $R_{\mathrm{ESR}}$ is the equivalent series resistance. $\mathrm{UC}_{i}$ can be the mass, to give the gravimetric energy/power density ( $\mathrm{W} \mathrm{h} \mathrm{kg} / \mathrm{W} \mathrm{kg}$ ), or the volume for the volumetric energy/power density ( $\mathrm{W} \mathrm{h} \mathrm{cm}^{-3} / \mathrm{W} \mathrm{cm}^{-3}$ ). In addition, cycle life is also an important parameter to evaluate the stability of a supercapacitor during repeated charge and discharge cycles.

For the flexibility evaluation of supercapacitors, no standardized or normalized evaluation method has been proposed to date. Nevertheless, those tests are often classified into three major forms: (1) bending test, (2) stretching test, (3) compressing test. Specifically, the CV curves and/or GCD curves are recorded under different bending/stretching/compressing states. The reversibility and mechanical strength tests after repeated bending/ stretching/compressing are alternative measures for flexibility evaluation of a supercapacitor.

Based on the above parameters, equations, and techniques, it is clear that $E_{\mathrm{i}}$ and $P_{\mathrm{i}}$ are highly dependent on the key parameters, $C_{\text {cell }}, V$, and $R_{\mathrm{ESR}}$. Therefore, great effort has been made to increase $C_{\text {cell }}$ and $V$ and to decrease $R_{\mathrm{ESR}}$. To enhance the flexibility of a supercapacitor, previous studies have focused on controlling the structural properties of the electrode materials and modifying the configuration of the supercapacitors. In the following sections, we will summarize the recent developments in flexible supercapacitors based on CNTs and graphene materials.

\section{CNTS-BASED FLEXIBLE SUPERCAPACITORS}

CNTs, which were first discovered in 1991 [37], have many unique properties such as high conductivity and good mechanical performance. For instance, an elastic modulus up to $1 \mathrm{TPa}$ and a tensile strength of $100 \mathrm{GPa}$ have been measured for an individual multiwall CNT (MWCNT) [38]. CNTs can be prepared via many processes such as chemical vapor deposition (CVD), catalytic pyrolysis, arc-discharge, and laser ablation [39]. Owing to the features mentioned above, CNTs are promising materials for use in flexible supercapacitors. However, the electrical properties of CNTs shift significantly with slight changes of chirality, diameter and wrapping angle $[39,40]$. As a result, CNTs are typically applied in the form of thin CNT films, aligned CNT arrays or interconnected CNT sponges, which may lead to radically different performance in flexible supercapacitors.

\section{CNT films}

Under controlled conditions, CNTs tend to interweave with each other to form a self-standing CNT thin films, which makes these films have a uniform distribution of electrical properties. Approaches including CVD/floating catalyst CVD [41-47], vacuum filtration [48-52], interfacial reaction [53,54], and printing [55] have been developed to fabricate CNT films for use in flexible supercapacitor applications.

Self-standing single wall CNT (SWCNT) films have been prepared by spreading out over a pre-strained polydimethylsiloxane (PDMS) substrate to obtain a continuous buckled SWCNT film when the stress was released [43] (Fig. 2a). The sheet resistance of the buckled film is almost constant even under $140 \%$ tensile strain. The specific capacitance without strain and under $120 \%$ strain were 48 and $53 \mathrm{~F} \mathrm{~g}^{-1}$, respectively, indicating good mechanical stability of the buckled CNT film. A vacuum filtration method was used to deposit SWCNT film onto the PDMS substrate to fabricate a transparent supercapacitor [50]. As shown in Fig. 2b, the device is highly transparent, and the optical transmittance is as high as $82 \%$. The obtained specific capacitance, maximum power and energy density are $34.2 \mathrm{~F} \mathrm{~g}^{-1}, 21.1 \mathrm{~kW} \mathrm{~kg}^{-1}$ and $18.0 \mathrm{~kW} \mathrm{~h} \mathrm{~kg}^{-1}$, respectively. A stretchable isotropic buckled CNT film exhibited omnidirectional stretch abilities in the range of $50 \%-200 \%$ by adjusting the prestrain applied to the CNT films (Fig. 2c-f). [45] The CNT film shows anisotropic elasticity and high endurance towards stretching when stretched in different directions (Fig. 2g). In addition, the resistance of the CNT film was mostly unchanged when axial stretching up to $200 \%$ was applied. The isotropic buckled CNT film only possesses a specific capacitance of $9.52 \mathrm{mF} \mathrm{cm}^{-2}$ but a much higher 

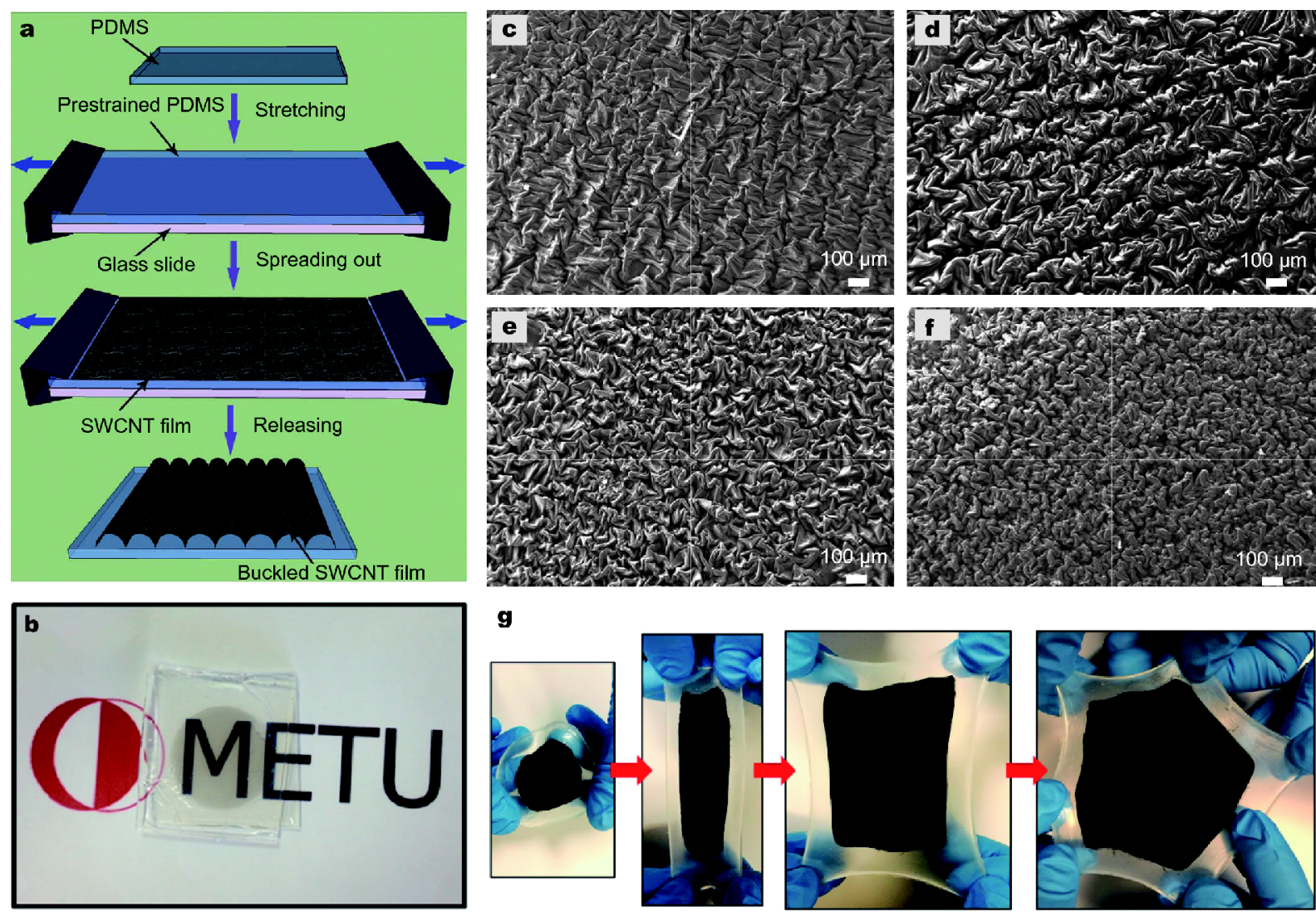

9
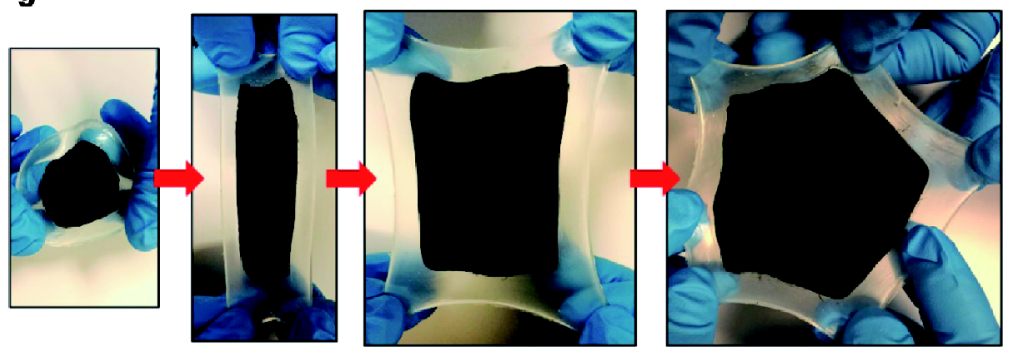

Figure 2 (a) Schematic illustration for preparing buckled SWCNT film on PDMS. Reproduced with permission from Ref. [43]. Copyright 2013, Wiley-VCH. (b) Photograph of the fabricated supercapacitors without carbon paste current collectors. Reproduced with permission from Ref. [50]. Copyright 2014, the American Chemical Society. SEM images of buckled structures formed in CNT films at different levels of omnidirectional prestrain (c) 50\%, (d) $100 \%$, (e) $150 \%$, and (f) $200 \%$. (g) Photos of buckled CNT film at various stretching states. Reproduced with permission from Ref. [45]. Copyright 2014, the American Chemical Society.

specific capacitance of $1147.52 \mathrm{mF} \mathrm{cm}^{-2}$ can be achieved when polyaniline (PANI) is electrodeposited onto the film surface.

To realize their wide applications in electronics and energy devices, it is critical to rationally assemble the carbon nanostructures into large-area films. Song et al. [56] reported a large area self-assembly method for preparing CNT films. First, CNTs were exposed to $\mathrm{HNO}_{3}$ vapor to increase the ratio of oxygen-containing groups. Next, the as-prepared CNTs were dispersed in water and a piece of copper foil was placed on the water surface. Metal-induced deoxygenation between the copper and oxidized CNTs leads to the coating of a dense black CNT film on the surface of the copper foil (Fig. 3a). The CNT film is easily transferred onto other substrates after etching the copper foil (Fig. 3b). For the bending test of the CNTs film on a paper, no conductivity degradation was observed after 300 bending cycles. To evaluate the electrochemical capacitive performance, a symmetric ca- pacitor was fabricated using the carbon film as the flexible electrodes, exhibiting an energy density of $3.5 \mathrm{~W} \mathrm{~h} \mathrm{~kg}^{-1}$ and a power density of $28.1 \mathrm{~kW} \mathrm{~kg}^{-1}$.

When flexible electronics are used in living tissues, compatibility must be considered. Recently, Shin et al. [55] reported a bioactive CNT-based ink for printing 2D and 3D flexible electronics. Deoxyribonucleic acid (DNA) was used as a natural surfactant to disperse the CNTs into various biomaterials, such as gelatin methacryloyl (GelMA) and hyaluronic acid (HA), to form bioactive CNTbased hybrid inks (Fig. 3c, d). Generally, the use of GelMA increases the resistance of the CNT junctions especially at high concentration. However, DNA can bind to the surface of the CNTs due to the strong $\pi-\pi$ interaction between the CNTs and the bases of the DNA segment. The hydrophilic phosphate groups on the DNA polymer are helpful to the dispersion of CNTs in the solution. Therefore, the dispersion of CNTs with a high concentration can be achieved by GelMA/DNA coating, re- 

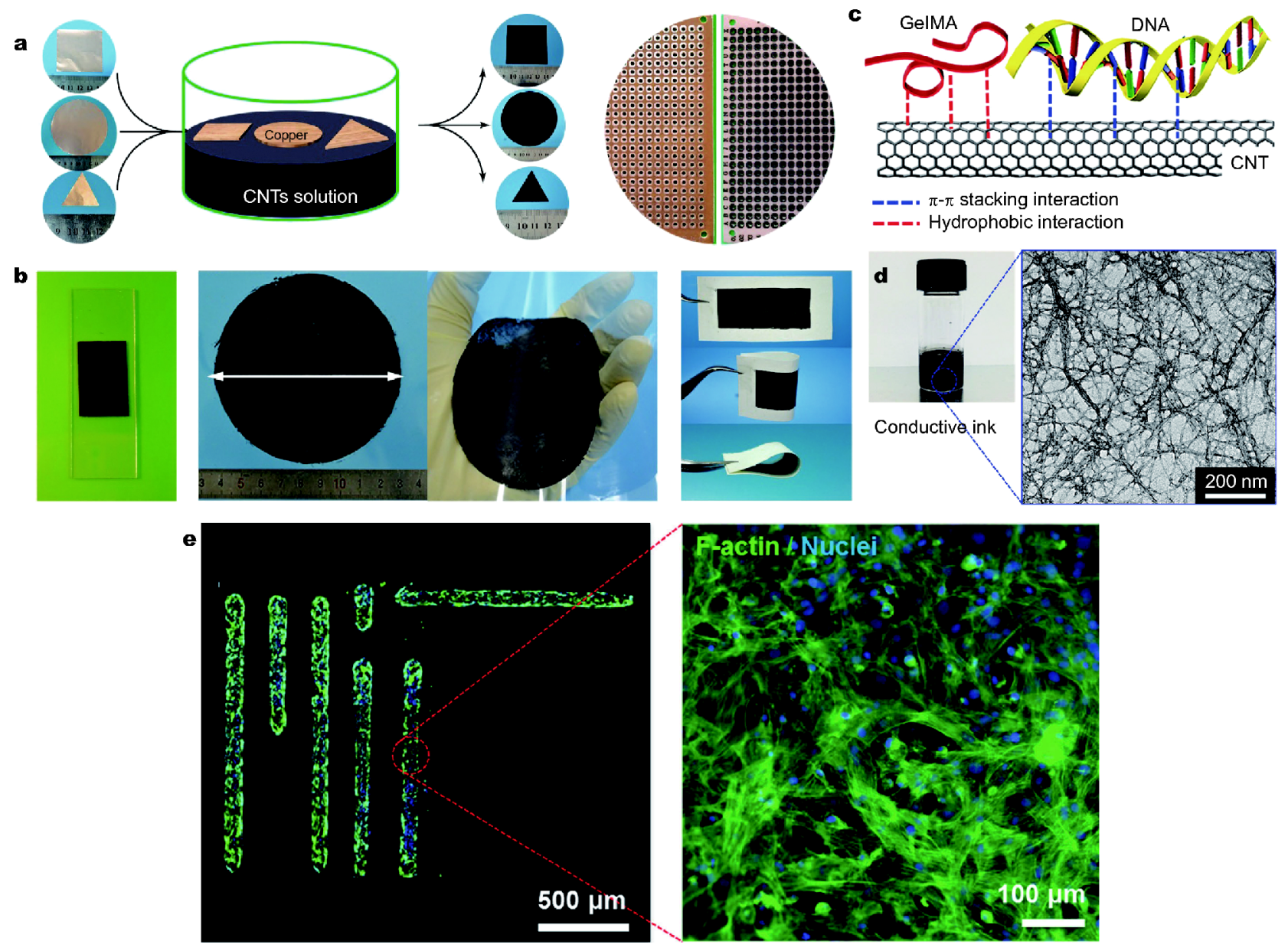

Figure 3 (a) Schematic illustration for the assembly of CNTs on copper and photographs of the copper foils before and after the deposition of CNTs. (b) SA-CNT films transferred to different substrates: glass (left), PET (middle), paper (right). Reproduced with permission from Ref. [56]. Copyright 2017, Wiley-VCH. (c) Schematic diagram showing the GelMA/DNA-coated MWCNT inks formed though hydrogen bonding, hydrophobic interactions, and $\pi-\pi$ stacking interactions between MWCNT and GelMA/DNA. (d) Photograph of black conductive ink and high-resolution TEM image of individual or bundled GelMA/DNA-wrapped MWCNTs. (e) Fluorescence images of cardiac fibroblasts cultured on ink patterns printed on PEGcoated PET film. F-actin and cell nuclei were immunostained and fluorescently labeled green and blue, respectively. Reproduced with permission from Ref. [55]. Copyright 2016, Wiley-VCH.

sulting in good electrical conductivity $\left(24 \pm 1.8 \mathrm{~S} \mathrm{~cm}^{-1}\right)$. Furthermore, the printed ink shows good cytocompatibility and cell adhesiveness (Fig. 3e). The seeded cardiac cell was only detected in the printed ink area and showed cellular viability of approximately $90 \%$ after 3 days. When tested in biological media, the ink-coated hydrogel had a specific capacitance of approximately $30 \mathrm{~F} \mathrm{~g}^{-1}$.

To enhance the capacitive performance, a large number of materials including polymers [41,44,52,54], metal oxides [47,51], metal sulfides [46], MXenes [49], and layered double hydroxides [48] have been combined with CNTs film. A facile method has been reported to fabricate MWCNT/PDMS films via a water surface assisted synthesis [53]. Typically, a mixture of MWCNT, xylol, silicone-elastomer base and the curing agent is poured on the surface of distilled water. Due to the insolubility and low density of the mixture, the polyaddition reaction of the silicone-elastomer will proceed on the water surface, leading to the formation of a MWCNT/PDMS thin film. This composite film can be sparated from the water surface, and can be stretched at a high strain of 50\% without degrading its conductivity $\left(\sim 4.19 \mathrm{~S} \mathrm{~cm}^{-1}\right)$ or structure. By adjusting the MWCNT content, the optimized composite film acted as an ideal current collector and mechanical support. After the electrodeposition of PANI, the MWCNT/PDMS/PANI electrode realized an areal capacitance of $481 \mathrm{mF} \mathrm{cm}^{-2}$ and a specific capacitance of $1023 \mathrm{~F} \mathrm{~g}^{-1}$. A solid-state supercapacitor (SSC) 

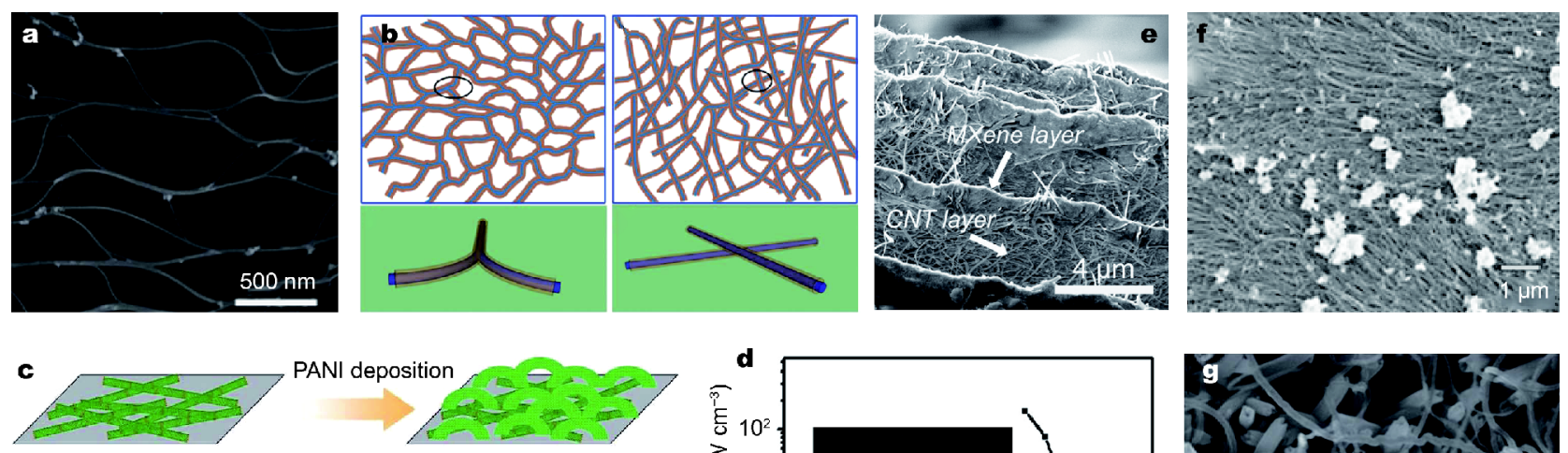

Electrochemical re-expanding
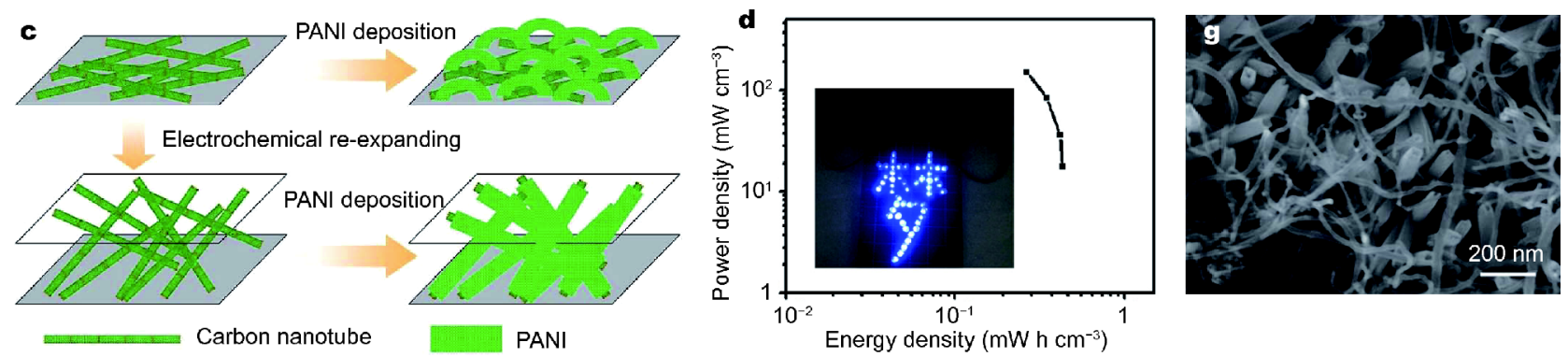

Figure 4 (a) SEM image of a single layer of SWCNT bundles peeled off from a thick film. (b) The sketch diagrams of a single layer film based on (left) continuous SWCNT/PANI reticulate structure and (right) randomly overlapped SWCNT/PANI bundles. "Blue" and "brown" parts represent SWCNT bundle skeleton and PANI skin. Reproduced with permission from [41]. Copyright 2012, the Royal Society of Chemistry. (c) Scheme of re-expanding process of CNT film. Reproduced with permission from Ref. [44]. Copyright 2015, the Royal Society of Chemistry. (d) Ragone plots of the supercapacity device (inset is 53 blue LEDs powered by a flexible SC pack consisting of 4 series-connected supercapacity devices). Reproduced with permission from Ref. [52] Copyright 2015, Elsevier. (e) Cross-sectional SEM images of $\mathrm{Ti}_{3} \mathrm{C}_{2} \mathrm{~T}_{x} / \mathrm{MWCNT}$ papers. Reproduced with permission from Ref. [49]. Copyright 2014, Wiley-VCH. (f) SEM images of aligned CNT/MoS $\mathrm{S}_{2}$ composite films with 3.1 wt.\% amounts of MoS . Reproduced with permission from Ref. [46]. Copyright 2016, Wiley-VCH. (g) Cross-sectional SEM image of the freestanding $\mathrm{CNT}_{\mathrm{MnO}} \mathrm{NT}_{\mathrm{N}}$ hybrid film. Reproduced with permission from Ref. [51]. Copyright 2014, the Royal Society of Chemistry.

was then assembled with two pieces of the MWCNT/ PDMS/PANI electrodes, an elastomeric polyurethane textile separator and $\mathrm{H}_{2} \mathrm{SO}_{4} / \mathrm{PVA}$ gel electrolyte. The asfabricated SSC showed good and stable capacitive behaviors under both static and dynamic stretching conditions. A similar interfacial reaction was used to generate flexible SWNT/PANI film at the chloroform/water interface [54]. Niu et al. [41] proposed a "skeleton/skin" strategy for preparing self-standing and flexible SWCNT/ PANI films. PANI was directly electrodeposited on the CNT films synthesized by a floating catalyst CVD method. The SEM image (Fig. 4a) of single layer SWCNT bundles peeled off from a thick film clearly illustrates that SWCNT bundles in the film are firmly bonded to form a continuous $2 \mathrm{D}$ reticulate structure. This unique structure allows electrons to be readily transported through the SWCNT network instead of PANI-PANI junctions (Fig. $4 \mathrm{~b})$, leading to the increased conductivity $\left(\sim 1148 \mathrm{~S} \mathrm{~cm}^{-1}\right)$. Flexible supercapacitor based on the SWCNT/PANI films exhibited high energy and power densities of 131 $\mathrm{W} \mathrm{h} \mathrm{kg}{ }^{-1}$ and $62.5 \mathrm{~kW} \mathrm{~kg}^{-1}$, respectively. A CNT/PANI hydrogel film was fabricated by an electrochemical reexpansion method [44]. During the re-expansion process, the CNT films prepared by floating catalyst CVD growth were activated by cyclic voltammetry scanning in a $\mathrm{H}_{2} \mathrm{SO}_{4}$ solution, leading to expansion of the film thickness from 0.01 to $1 \mathrm{~mm}$. As shown in Fig. 4c, this process can increase the internal space of the dense CNT film, benefiting the subsequent electrodeposition of PANI. In comparison with pristine CNT film decorated with PANI, the electrochemically activated CNT film with PANI coating exhibited a higher specific area capacitance of $680 \mathrm{mF} \mathrm{cm}^{-2}$. Polypyrrole (PPy) has also been electrodeposited onto vacuum filtrated CNTs films [52]. The flexible supercapacitor fabricated with CNT/PPy electrodes delivered an areal capacitance of $0.28 \mathrm{~F} \mathrm{~cm}^{-2}$ and could power 53 LEDs (Fig. 4d).

MXenes are a novel family of 2D metal carbides materials, which shows great potential in the applications of Li-ion batteries and supercapacitors. However, the restacking of MXene sheets limits the diffusion of electrolyte ions, seriously hindering their large-scale application. To address this issue, an alternating filtration method was used to fabricate sandwich-like $\mathrm{Ti}_{3} \mathrm{C}_{2} \mathrm{~T}_{x} /$ MWCNT papers [49]. In brief, dispersions of $\mathrm{Ti}_{3} \mathrm{C}_{2} \mathrm{~T}_{x}$ and MWCNT were filtrated using a polypropylene membrane in sequence to 
yield composite films composed of alternating $\mathrm{Ti}_{3} \mathrm{C}_{2} \mathrm{~T}_{x}$ and MWCNTs layers. This layer by layer structure (Fig. 4e) exhibits higher conductivity $\left(\sim 230 \mathrm{~S} \mathrm{~cm}^{-1}\right)$ than the randomly mixed $\mathrm{Ti}_{3} \mathrm{C}_{2} \mathrm{~T}_{x} /$ MWCNTs paper $\left(\sim 189 \mathrm{~S} \mathrm{~cm}^{-1}\right)$. When used as electrodes for supercapacitors, a specific capacitance of $150 \mathrm{~F} \mathrm{~g}^{-1}$ could be achieved, which was approximately $130 \%$ higher than the pure $\mathrm{Ti}_{3} \mathrm{C}_{2} \mathrm{~T}_{x}$. When the scan rate increased from 2 to $200 \mathrm{mV} \mathrm{s}^{-1}$, a capacitive retention of approximately $78 \%$ was observed, suggesting a good high-rate performance. Lv et al. [46] assembled a highly stretchable supercapacitor based on aligned CNTs and molybdenum disulfide $\left(\mathrm{MoS}_{2}\right)$. Different from the above mentioned CNT films, this vertically aligned CNT array was pressed against an elastic PDMS film, resulting in the formation of horizontally aligned CNT films, followed by $\mathrm{MoS}_{2}$ coating on by the drop-casting method. In the composite film, the CNTs successfully maintain their horizontal alignment (Fig. 4f), which resulted in the efficient transportation of electrolytes into the inner surface of the electrode for improved supercapacitor perfornace. The assembled supercapacitor based on the $\mathrm{CNT} / \mathrm{MoS}_{2}$ composite exhibited good flexibility upon bending or twisting and could be stretched up to $240 \%$. Moreover, $96 \%$ and $91 \%$ of the original supercapacitance was retained after being stretched to strains of $100 \%$ and $160 \%$, respectively for 500 cycles. As a representative example, homogeneous dispersions of manganese dioxide $\left(\mathrm{MnO}_{2}\right)$ nanotubes and CNTs were prepared in the presence of sodium dodecylbenzenesulfonate, and subsequent vacuum filtration of the homogeneous dispersion formed a free-standing $\mathrm{MnO}_{2} / \mathrm{CNTs}$ composite film [51]. The cross-sectional SEM images (Fig. 4g) show the good homogeneity of the as-prepared film. Two $\mathrm{MnO}_{2} / \mathrm{CNTs}$ film electrodes were incorporated into a flexible solidstate supercapacitor, which delivered a volumetric capacitance of $5.1 \mathrm{~F} \mathrm{~cm}^{-3}$ and an energy density of $0.45 \mathrm{~mW} \mathrm{~h} \mathrm{~cm}^{-3}$. Metal oxides and polymers combined to give a synergistic effects, and following this logic, Li et al. [47] fabricated a flexible symmetric supercapacitor based on $\mathrm{Fe}_{3} \mathrm{O}_{4} / \mathrm{CNT} /$ polyaniline ternary electrodes, exhibiting an energy density of $28.0 \mathrm{~W} \mathrm{~h} \mathrm{~kg}^{-1}$ and a power density of $5.3 \mathrm{~kW} \mathrm{~kg}^{-1}$. In the ternary electrode with a high electrical conductivity of $\sim 6800 \mathrm{~S} \mathrm{~cm}^{-1}$, the CNTs act as the flexible current collector and substrate while PANI serves as a protective shell to improve the structural stability of the $\mathrm{Fe}_{3} \mathrm{O}_{4}$ particles. PANI and CNTs also enhance the Faradaic processes across the interface, allowing the ternary electrode to take advantage of the high capacitance originating from the surface of the $\mathrm{Fe}_{3} \mathrm{O}_{4}$ particles and of the effective pseudocapacitance generated by PANI. A digital watch can be powered for 9 min with four such devices connected in series. A foldable bifunctional integrated device was fabricated by using SWCNT films and $\mathrm{TiO}_{2}$ nanoparticles (NPs) [57]. In that study, SWCNTs were synthesized with the floating catalyst CVD method and spread on both sides of a cellulose fiber paper. Then, a $\mathrm{TiO}_{2} \mathrm{NP}$ suspension was dripped onto one side of the cellulose paper, and PVA/LiCl electrolyte was dripped onto the other side of the paper, and all-solid-state integrated supercapacitor-photodetectors were obtained after drying. Upon irradiation with white light, the $\mathrm{TiO}_{2}$ NPs allow to yield electrons and holes. When an external field resulting from the charged supercapacitor is applied, the electron-hole pairs are separated. The electrons then move towards the positive electrode while the holes move to the negative electrode. As a result, the leakage current of the supercapacitor is enhanced and white light can be detected by the change in leakage current. This bifunctional device demonstrated a specific capacitance of $28 \mathrm{~F} \mathrm{~g}^{-1}$ and sensitivities of 24.7 and 38.5 under white and UV light, respectively.

\section{CNT arrays}

Owing to the random aggregation of CNTs commonly observed in typical solution processing, charges have to cross many boundaries in random CNT networks with low transfer efficiency, resulting in poor electrochemical performance of the CNTs [58-66]. Aligned carbon nanotubes, called as carbon nanotube arrays, have excellent electrical conductivity and provide an outstanding charge transfer rate [63]. To fully utilize these electroactive materials, the main strategy has been to maximize the active surface area while maintaining the high electric conductivity with the construction of a 3D nanostructure being the most common method to achieve these goals. Recently, CNTs grown on aluminum foil using a CVD method were directly incorporated into devices as the working electrode for the electrodeposition of PANI [64]. Interestingly, it was found that the distance between two aligned CNTs determined the specific capacitance because it influenced the full utilization of PANI and had significant effects on the diffusion of ions. After electrodeposition, the PANI coated CNTs on Al foil were cut into two pieces to fabricate a flexible supercapacitor using a filtration paper as the separator. (Fig. 5a) The composite materials exhibited a specific capacitance of $705.8 \mathrm{~F} \mathrm{~g}^{-1}$ at a discharge rate of $0.1 \mathrm{~A} \mathrm{~g}^{-1}$. A transparent and flexible MWCNT/PANI film was synthesized by Lin et al. [65] which featured aligned MWCNT sheets prepared by drydrawing MWCNT arrays and showed high electrical 

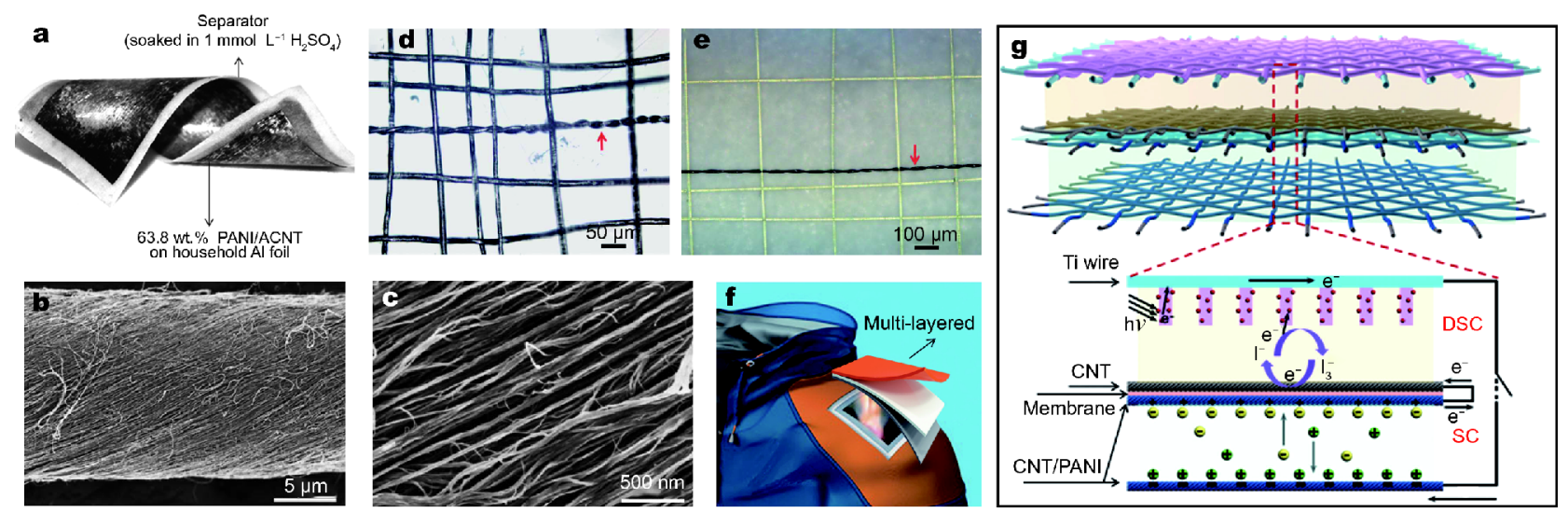

Figure 5 (a) Digital photograph of the flexible symmetric supercapacitor with a filtration paper as separator. Reproduced with permission from Ref. [64]. Copyright 2012, Wiley-VCH. SEM images of bare aligned MWCNTs at low (b) and high (c) resolution. The supercapacitor wire woven into other CNT fibers (d) and a textile composed of aramid fibers (e). The red arrows show the wire. Reproduced with permission from Ref. [62]. Copyright 2013, the Royal Society of Chemistry. (f) Photograph of multilayered clothes. (g) Schematic illustration to the integrated energy textile. The enlarged view shows the working mechanism. Reproduced with permission from Ref. [67]. Copyright 2014, Wiley-VCH.

conductivity of $570 \mathrm{~S} \mathrm{~cm}^{-1}$. The sheets functioned as effective electrodes for the electrodeposition of PANI and the flexible supercapacitor based on the transparent film had a specific capacitance of $233 \mathrm{~F} \mathrm{~g}^{-1}$ at a current density of $1 \mathrm{~A} \mathrm{~g}^{-1}$. In addition, the values of specific capacitances remain unchanged when the bending angle of the supercapacitor increased from 0 to $180^{\circ}$. A weavable wireshaped microsupercapacitor has also been fabricated using PANI incorporated aligned MWCNT composite fibers [62]. SEM images (Fig. 5b, c) show that the CNT fibers could be spun from the MWCNT arrays and maintained the aligned structure. The CNT fibers possessed good tensile strength of $10^{2}-10^{3} \mathrm{MPa}$ and conductivity of $10^{3} \mathrm{~S} \mathrm{~cm}^{-1}$. The uniform coating of PANI on the surface of the MWCNT fibers further improved the tensile strength by $58 \%$ without losing electrical conductivity. Two composite fibers were twisted together to fabricate a wire-shaped microsupercapacitor, which delivered a specific capacitance of $274 \mathrm{~F} \mathrm{~g}^{-1}$. As shown in Fig. $5 \mathrm{~d}$, e, the wire-shape supercapacitor can easily be woven into other CNT fibers or textiles, and the aligned CNT array fibers coated with PANI have been woven into textiles [67]. The thin, transparent and flexible supercapacitor textile was further integrated with a photoelectric conversion (PC) device, with the goal of directly storing the electric energy generated by a solar cell in a single device. First, a titanium wire textile grown with perpendicularly aligned $\mathrm{TiO}_{2}$ nanotubes was covered with a CNT-fiber textile using a gel electrolyte and 3-methoxypropionitrile solution containing $0.1 \mathrm{~mol} \mathrm{~L}^{-1} \mathrm{LiI}, 0.05$ $\mathrm{mol} \mathrm{L}^{-1} \mathrm{I}_{2}, 0.5 \mathrm{~mol} \mathrm{~L}^{-1}$ 4-tert-butylpyridine and 0.5 mol L ${ }^{-1}$ 1-propyl-2,3-dimethylimidazolium iodide. Then, the PC was stacked with the supercapacitor textile with a membrane separating the two (Fig. $5 \mathrm{~g}$ ). This prototype device can realize a photoelectric conversion and storage efficiency of $1.5 \%$, which indicates great potential for application in multilayered clothes (Fig. 5f).

Due to their low cost and potential for scalable production of carbon materials, combining different carbon structures also has attracted significant attention. To combine the high porosity and better ion diffusion properties of CNT array with the superior mechanical properties and high electrical conductivity of CNT sheets, Malik et al. [68] used a plasma enhanced CVD method to directly grow CNT arrays on a CNT sheet, leading to the formation of a 3D structure comprised of vertically aligned CNTs extending from horizontally aligned CNTs in the CNT sheet substrates. Then, controlled PANI deposition was achieved via cyclic voltammetry methods, maintaining the novel 3D nanostructure (Fig. 6a, b) during and after the electrodeposition of PANI. Moreover, nitrogen doping of CNT arrays was successfully performed during the CNT growth process, which further improves the electrochemical performance of CNT arrays. When assembled into a flexible symmetric supercapacitor, the electrodes demonstrate a gravimetric capacitance of $359 \mathrm{Fg}^{-1}$ at a current density of $1.56 \mathrm{~mA} \mathrm{~cm}^{-2}$. Cherusseri et al. [69] assembled a flexible fibrous supercapacitor based on oxidized CNT (OCNT)/ PPy brush-like electrodes. Initially, carbon fibers were coated with nickel through an electroless bath method. CNTs grew on the nickel coated carbon fibers (CFs) by 

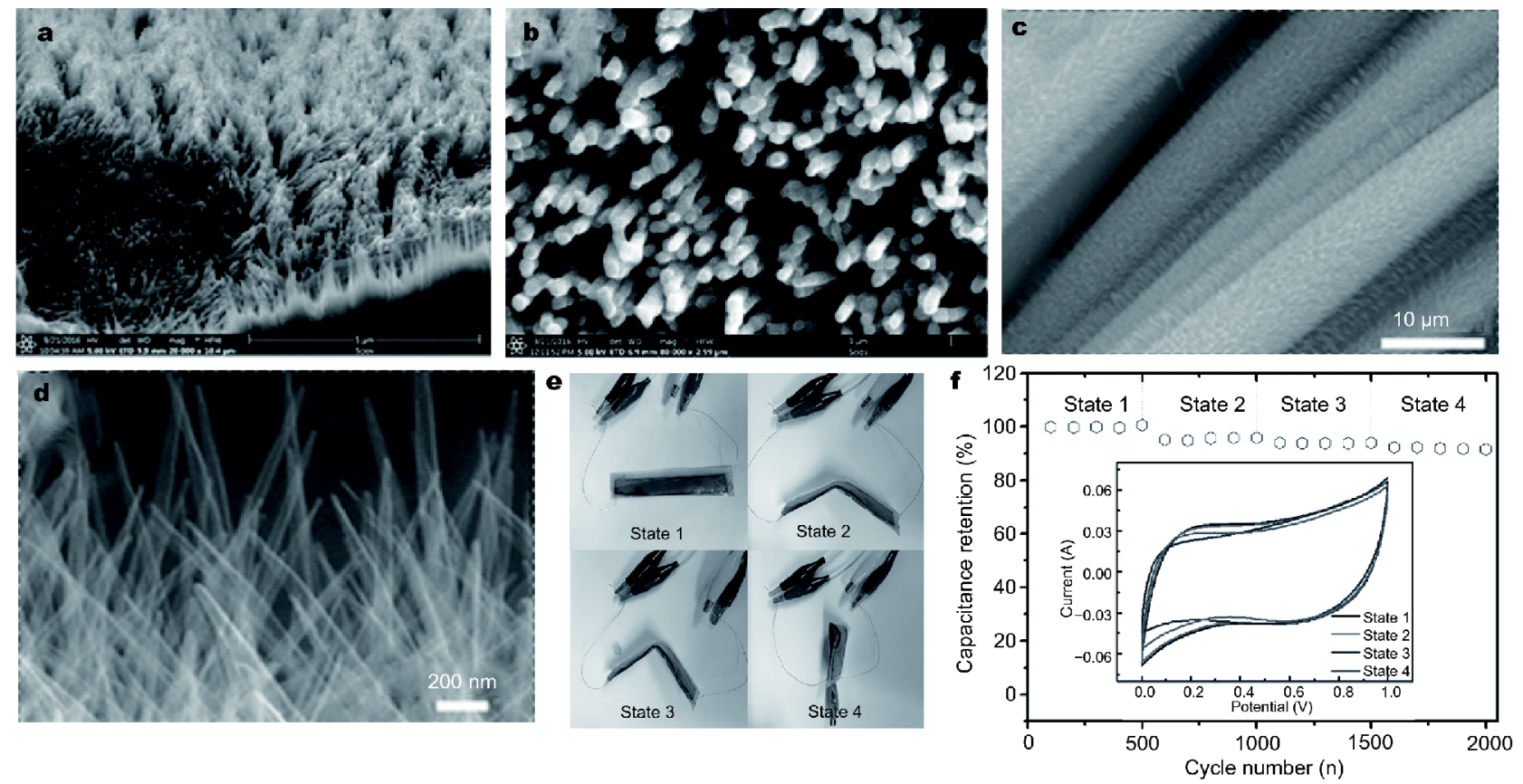

Figure 6 SEM images of CNT arrays before (a) and after (b) PANI deposition. Reproduced with permission from Ref. [68] Copyright 2017, Elsevier. Low (c) and high (d) magnification SEM images of the PCNTAs@CFs. (e) Photographs of PCNTAs@CFs symmetric device at different bending states. (f) Cycle stability tested at different bending states with the inset showing CV curves obtained at different bending states at a scan rate of $500 \mathrm{mV} \mathrm{s}{ }^{-1}$. Reproduced with permission from Ref. [70]. Copyright 2016, Wiley-VCH.

the CVD method and converted into OCNT after annealing in an air-bed reactor. Then, polypyrrole was deposited on the brush-like electrodes via a constant current method. The obtained composite electrode were used to fabricate a flexible supercapacitor, exhibiting a gravimetric capacitance of $305 \mathrm{~F} \mathrm{~g}^{-1}$ and an energy density of $42 \mathrm{~W} \mathrm{~h} \mathrm{~kg}^{-1}$. X-ray photoelectron spectroscopy (XPS) and Fourier transform infrared (FTIR) analyses revealed that the surface functional groups of CNT contribute to maximize the pseudo-Faradaic charge storage. Similarly, a novel and scalable synthetic approach was been developed to produce hierarchically aligned porous CNT arrays (PCNTAs) on the flexible CFs (PCNTA@CFs) [70]. $\mathrm{ZnO}$ nanorod arrays on CFs synthesized hydrothermally were used as templates for the growth of PCNTAs. Following catalytic conversion of ethanol on the $\mathrm{ZnO}$ nanorod arrays, the removal of the $\mathrm{ZnO}$ templates led to the formation of the final PCNTAs@CF material. The SEM images of the PCNTAs@CF in Fig. 6c, d show the ideal hierarchical carbon nanostructure. The high-aspect-ratio enabled the large surface-to-volume ratio, which ensures a large ion-accessible surface area. To investigate the flexibility of the PCNTAs@CF electrodes in a symmetric supercapacitor, $\mathrm{CV}$ tests were performed under different bending states. Almost no change was observed in the CV curves (Fig. 6e, f), indicating good flexibility and mechanical stability of the device. As summarized above, aligned CNTs are good substrates for constructing flexible supercapacitors due to their good mechanical stability and high conductivity. Hybrid fibers composed of vertically aligned SWCNTs and reduced graphene oxide (RGO) were successfully constructed via a hydrothermal method with ethylenediamine (EDA) as a nitrogen dopant and fused silica capillary columns as microreactors [71]. The EDA molecule with a $-\mathrm{NH}_{2}$ end group can act as an "end-anchoring" reagent to bind the acid-oxidized SWNTs and GO via self-assembly process. During the hydrothermal process, nitrogen doping improved the surface conductivity and wettability and induces pseudocapacitance in carbon nanomaterials. The hybrid fiber containing mesoporous structures had a large specific surface area of $\sim 396 \mathrm{~m}^{2} \mathrm{~g}^{-1}$ and a high electrical conductivity of $\sim 102 \mathrm{~S} \mathrm{~cm}^{-1}$. An all-solid-state micro-supercapacitor made from the hybrid fiber electrodes on a flexible polymeric substrate exhibited a volumetric capacitance of $300 \mathrm{~F} \mathrm{~cm}^{-3}$ and it retained more than $97 \%$ of its initial capacitance after bending 1000 times at $90^{\circ}$. Controlling the morphology and porosity is two important strategies for improving the electrochemical performance of aligned CNTs. 

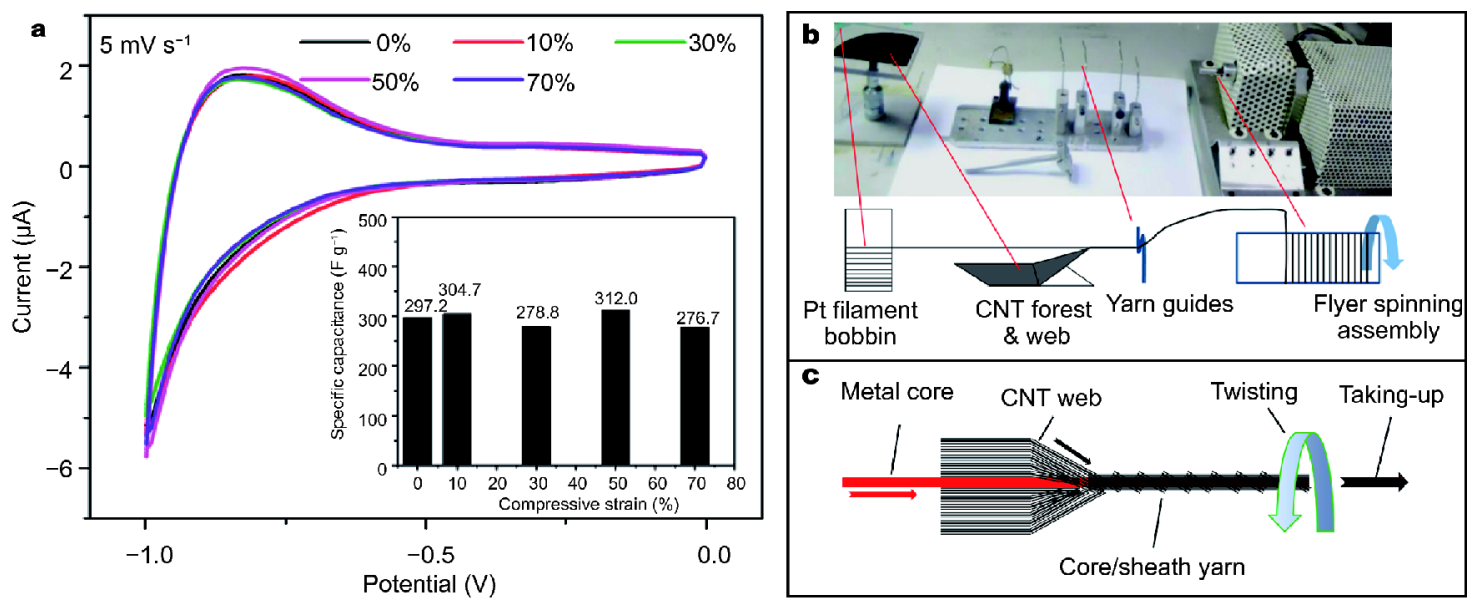

Figure 7 (a) CV curves of the $\mathrm{CNT} @ \mathrm{Fe}_{2} \mathrm{O}_{3}$ sponge under different compressed conditions $(0,10 \%, 30 \%, 50 \%$ and $70 \%)$ at $5 \mathrm{mV} \mathrm{s}$. The inset shows the specific capacitance of the samples under different compressive strains at a scan rate of $5 \mathrm{mV} \mathrm{s} \mathrm{s}^{-1}$. Reproduced with permission from Ref. [74]. Copyright 2015, the Royal Society of Chemistry. (b) Continuous spinning of Pt/CNT core/sheath yarns: photograph of the flyer spinner and schematics of working elements. (c) Schematic showing formation of the core/sheath yarn structure. Reproduced with permission from Ref. [77]. Copyright 2014, the American Chemical Society.

\section{Other CNT nanostructures}

In addition to CNT film and arrays, other CNT nanostructures have also been developed for use in flexible supercapacitors, such as CNT networks [72], CNT sponges [73-75], and CNT yarn [76-78]. These CNT structures are often used as conductive skeletons/substrates, and provide a large surface area for the deposition of secondary active materials by following the so-called core-sheath/shell model. To achieve maximum electroactive surface area, the structure of the sheath layer must be carefully regulated rather than just simply coated onto the CNT core. Accordingly, Chen and co-workers prepared a 3D $\quad \alpha-\mathrm{Fe}_{2} \mathrm{O}_{3} /$ carbon nanotube $\left(\mathrm{CNT} @ \mathrm{Fe}_{2} \mathrm{O}_{3}\right)$ sponge electrode with a hierarchical structure, consisting of a compressible and conductive CNT network coated with a layer of $\mathrm{Fe}_{2} \mathrm{O}_{3}$ nanohorns [74]. First, $\mathrm{FeOOH}$ was uniformly deposited onto a CNT sponge by a hydrothermal method and then transformed into spindle-like $\mathrm{Fe}_{2} \mathrm{O}_{3}$ crystals in the subsequent annealing process. The obtained CNT@ $\mathrm{Fe}_{2} \mathrm{O}_{3}$ exhibited a good tolerance towards compression. The CV curves (Fig. 7a) of five CNT@ $\mathrm{Fe}_{2} \mathrm{O}_{3}$ electrodes show only small differences when compressed with strains of $0,10 \%, 30 \%, 50 \%$ and $70 \%$. After 1000 compression cycles, the $\mathrm{CV}$ curves of the electrode showed negligible change, indicating that the 3D CNT hybrid sponge could withstand compression and function as a stable electrode under the repeated compression cycles. Compared with a pure CNT sponge, the $\mathrm{CNT} @ \mathrm{Fe}_{2} \mathrm{O}_{3}$ sponge exhibited enhanced specific capacitance of $296.3 \mathrm{~F} \mathrm{~g}^{-1}$ due to the reversible redox reaction of $\mathrm{Fe}_{2} \mathrm{O}_{3}$ during the charge/discharge process. Li et al. [79] reported a core/double-shell structure of the CNT@PPy@MnO 2 sponge. In the porous hierarchical structure, the CNT sponge acts as a conductive skeleton while PPy is an intermediate layer that improves the interaction between the CNT and $\mathrm{MnO}_{2}$ layers. As the outside layer, $\mathrm{MnO}_{2}$ is able to enhance the capacitive performance due to the redox reactions. It was found that the sequence of the shells has a significant influence on the capacitive performance of the device. The hybrid

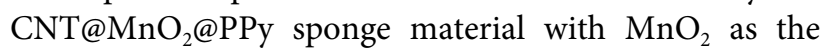
intermediate layer exhibited a lower specific capacitance of $241 \mathrm{~F} \mathrm{~g}^{-1}$ than that of the CNT@PPy@MnO $\left(325 \mathrm{~F} \mathrm{~g}^{-1}\right)$. The charge transfer resistance, $R_{\mathrm{ct}}$, of the CNT@MnO $\mathrm{MPPy}_{2}$ sponge was also much higher than that of the CNT@PPy@MnO ${ }_{2}$ sponge. These results show that the PPy intermediate layer was effective in reducing the interfacial resistance and improving the charge transfer between the $\mathrm{CNT}$ and $\mathrm{MnO}_{2}$ layers. Furthermore, this core/double-shell sponge can maintain its open porous and stable structure under high compressive strains. Thus, no obvious current change was observed even at $50 \%$ compression.

In flexible supercapacitors, CNT yarn can be used as a multifunctional active material and current collector. CNT yarn can also be bound on a linear substrate such as metal wires. Zhang et al. [77] reported a core/spun CNT yarn supercapacitor for use in wearable electronic textiles. On a flyer spinner (Fig. 7b), a continuously rolling platinum monofilament was pulled from the bobbin on the left side to the collection bobbin on the right side. At the same time, CNTs are drawn from the MWCNT forest to 
form a continuous sheath of yarn due to the strong $\pi-\pi$ interactions among carbon nanotubes. During this process, CNTs were rotated together with a Pt filament and wrapped on the filament to form a core/sheath-structured yarn (Fig. 7c). The resultant core/sheath yarn with a diameter of $29.5 \mu \mathrm{m}$ had a high tensile strength of $550 \mathrm{MPa}$, indicating that the CNT yarn can be woven into thinner fabrics, comparable to traditional textiles, while possessing higher tensile strength. The $\mathrm{Pt} / \mathrm{CNT}$ yarn was further coated with PANI and PVA- $\mathrm{H}_{2} \mathrm{SO}_{4}$ electrolyte to fabricate a two-ply yarn supercapacitor. In the investigation of the flexibility of the obtained supercapacitors, only slight changes were observed in the capacitance after 1000 cycles of folding and unfolding, indicating that the two-ply yarn supercapacitors are a potential power source in wearable electronics..

\section{GRAPHENE-BASED FLEXIBLE SUPERCAPACITORS}

Due to its extraordinary mechanical strength, high surface area, as well as good electrical and thermal conductivity, graphene has become one of the most promising candidates in supercapacitor applications [2830]. However, the above-mentioned characteristics are influenced by many parameters such as the stacking layer numbers and the morphology of the constituent graphene. Over the last few years, various graphene materials including graphene fibers, graphene films, and 3D porous graphene frameworks have been synthesized to improve supercapacitors' performance. The electrochemical performances of some graphene-based flexible supercapacitors are summarized in Table 1.

\section{Graphene fibers}

Graphene fibers can be synthesized through hydrothermal [80], wet spinning [81], and self-assembly [82] processes. Because of their mechanical stability and good conductivity, graphene fibers can be easily woven or knitted into textiles. By controlling the evaporation of solvents, graphene films grown by CVD could be selfassembled into fibers with designated morphology and pore structure [83]. The obtained graphene fiber showed an areal capacitance of $1.4 \mathrm{mF} \mathrm{cm}$ in $\mathrm{Na}_{2} \mathrm{SO}_{4}$ aqueous solution. Due to its low specific surface area and poor hydrophilicity, pure graphene fibers usually exhibit only a small capacitance $[82,84]$. To increase the specific capacitance of graphene fiber based materials, approaches to enlarge the surface area and/or incorporate with pseudocapacitive materials have been developed. Meng et al. [84] first synthesized graphene fiber using a hydrothermal method, and then RGO was deposited onto the graphene fiber to form a 3D porous network-like graphene layer via an electrolyzing process. During this process, GO sheets were deposited around the graphene fiber core and were reduced to the conductive RGO. As shown in Fig. 8a, the dense graphene fiber core surrounded by porous RGO sheets exhibits a typical core/sheath structure. The measured length-specific, area-specific, and mass-specific capacitances can reach $20 \mu \mathrm{F} \mathrm{cm}^{-1}, 1.2-1.7 \mathrm{mF} \mathrm{cm}^{-2}$ and 25-40 $\mathrm{F} \mathrm{g}^{-1}$, respectively. After weaving into a textile, the fiber supercapacitor maintained its flexibility and no capacitance loss was observed while bending (Fig. $8 \mathrm{~b}-\mathrm{d}$ ). A similar electrolyzing process was performed on a gold wire to deposit RGO sheets. The fabricated fiber-shaped supercapacitor exhibited a capacitance of $11.4 \mu \mathrm{F} \mathrm{cm}$ [85]. A well-controlled laser reduction was used to reduce the upper and lower sides of a fresh GO fiber under laser irradiation, leading to the formation of a RGO-GO-RGO fiber [86]. In the prepared RGO-GO-RGO fiber, the RGO on the upper and lower sides act as electrodes while the GO layer serves as the separator as well as the electrolyte container simultaneously. Thus, a fiber supercapacitor was achieved when the RGO sides were connected to the external circuit by two gold sheets (Fig. 8e). The energy and power densities of the all-in-one fiber capacitors were $2-5.4 \times 10^{-4} \mathrm{~W} \mathrm{~h} \mathrm{~cm}^{-2}$ and $3.6-9 \times 10^{-2} \mathrm{~W} \mathrm{~cm}^{-2}$, respectively, comparable to carbon-based and metal oxide supercapacitors [87,88]. A scalable non-liquid-crystal (NLC) spinning method was developed [89] to generate locally aligned RGO fibers for fabricating wearable supercapacitors. Compared with graphene fibers obtained from wet-spinning of liquid-crystal (LC) GO dispersions, the fibers generated from an NLC GO dispersion (Fig. 9a, b) have a relatively smooth surface at the microscale but exhibit a bark-like structure at the nanoscale. This unique structure facilitates the diffusion of electrolyte ions due to its bark-like surface morphology and the locally aligned pore structure of the NLC-spun RGO fibers. When immersed in a $1 \mathrm{~mol} \mathrm{~L}^{-1} \mathrm{H}_{2} \mathrm{SO}_{4}$ electrolyte, the calculated mass-specific and volume-specific capacitances was $279 \mathrm{~F} \mathrm{~g}^{-1}$ and $340 \mathrm{~F} \mathrm{~cm}^{-3}$, respectively, at a current density of $0.2 \mathrm{~A} \mathrm{~g}^{-1}$. To fabricate a prototype of wearable supercapacitors, three solid-state yarn supercapacitors connected in series were woven into a piece of cloth (Fig. 9c). This prototype device was able to light up a red LED for 5 min while maintaining the flexibility of the original cloth. CNTs can also be used as additives to increase the mechanical strength and conductivity of graphene fibers. Graphene fibers intercalated with $\mathrm{Fe}_{3} \mathrm{O}_{4}$ nanoparticles were synthesized through heat treatment in a glass pi- 


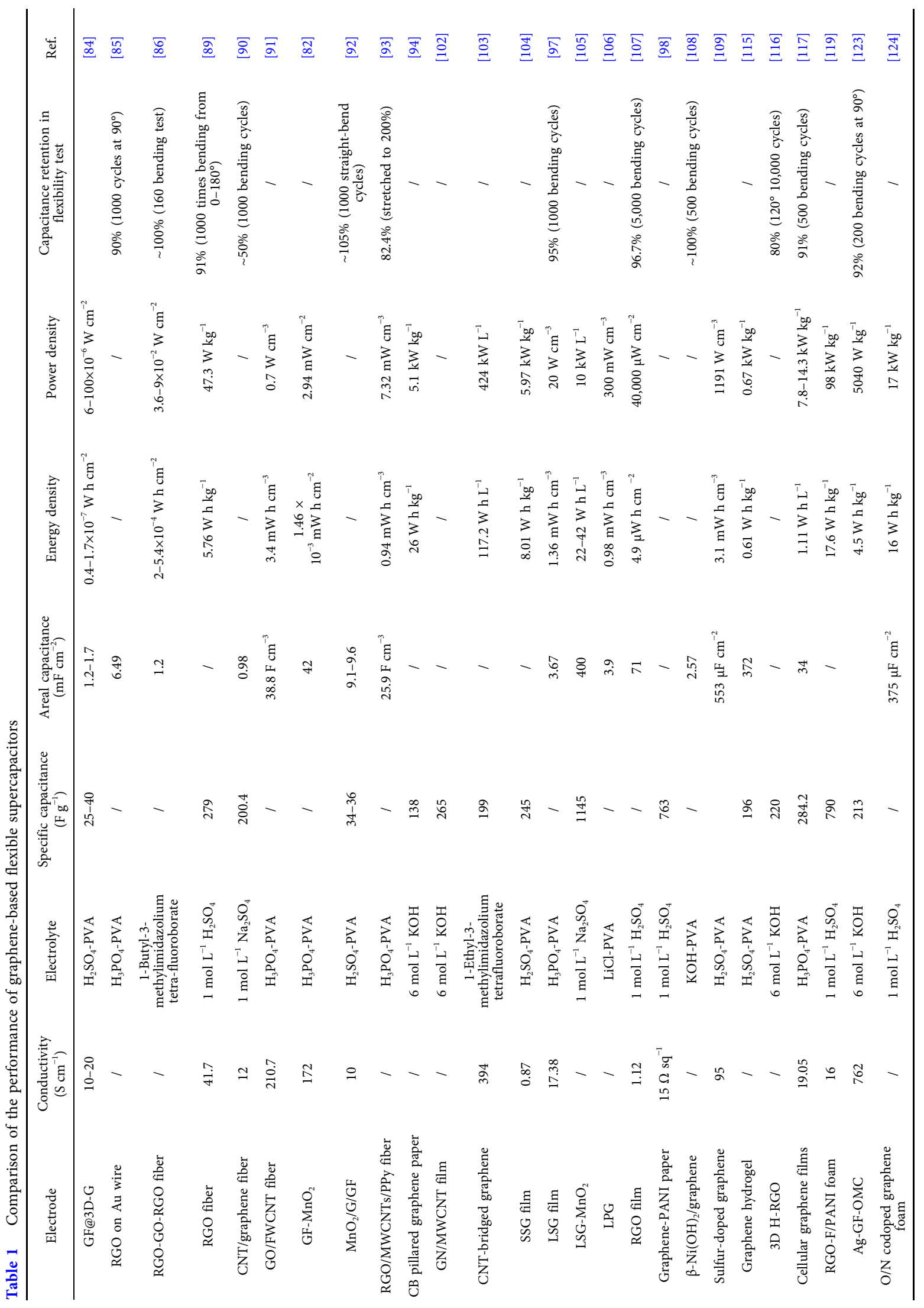



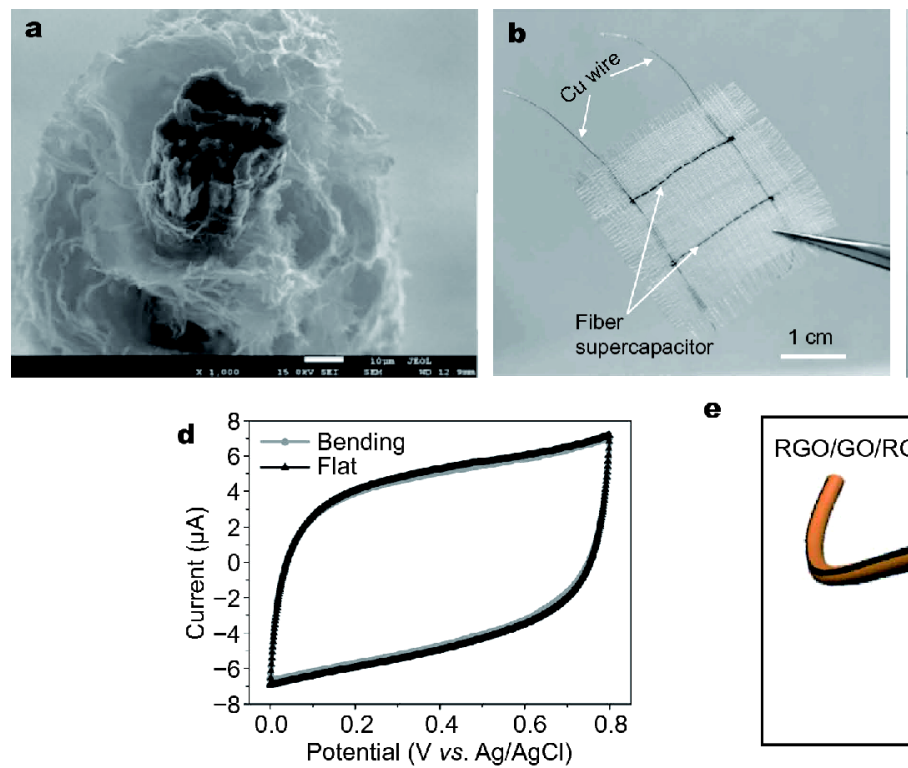

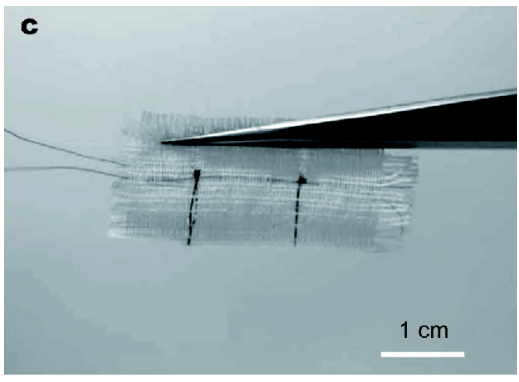

$\mathbf{e}$

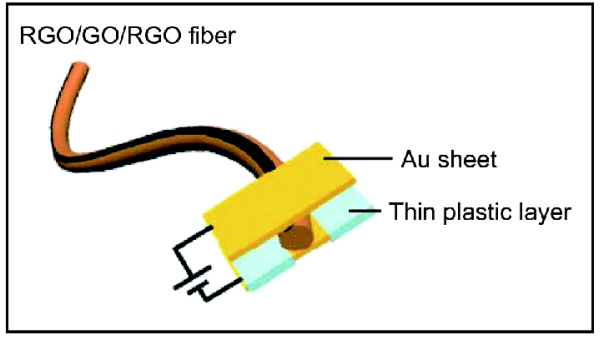

Figure 8 (a) Cross-section view of a GF@3D-G showing the core GF surrounding with standing graphene sheets. Photos of the textile embedded with two GF@3D-G fiber supercapacitors in flat (b) and bending (c) state, respectively. (d) CV curves of two GF@3D-G fiber supercapacitors as the textile in flat (b) and bending (c) states with a scan rate of $50 \mathrm{mV} \mathrm{s}^{-1}$. Reproduced with permission from Ref. [84]. Copyright 2013, Wiley-VCH. (e) The scheme of the RGO-GO-RGO supercapacitor device, where RGO layers were in contact with the Au sheets. The thin plastic layer of polyvinylidene chloride (PVDC) was partially coated on the Au surface to prevent the short circuit of two Au electrodes. Reproduced with permission from Ref. [86]. Copyright 2014, the Royal Society of Chemistry.
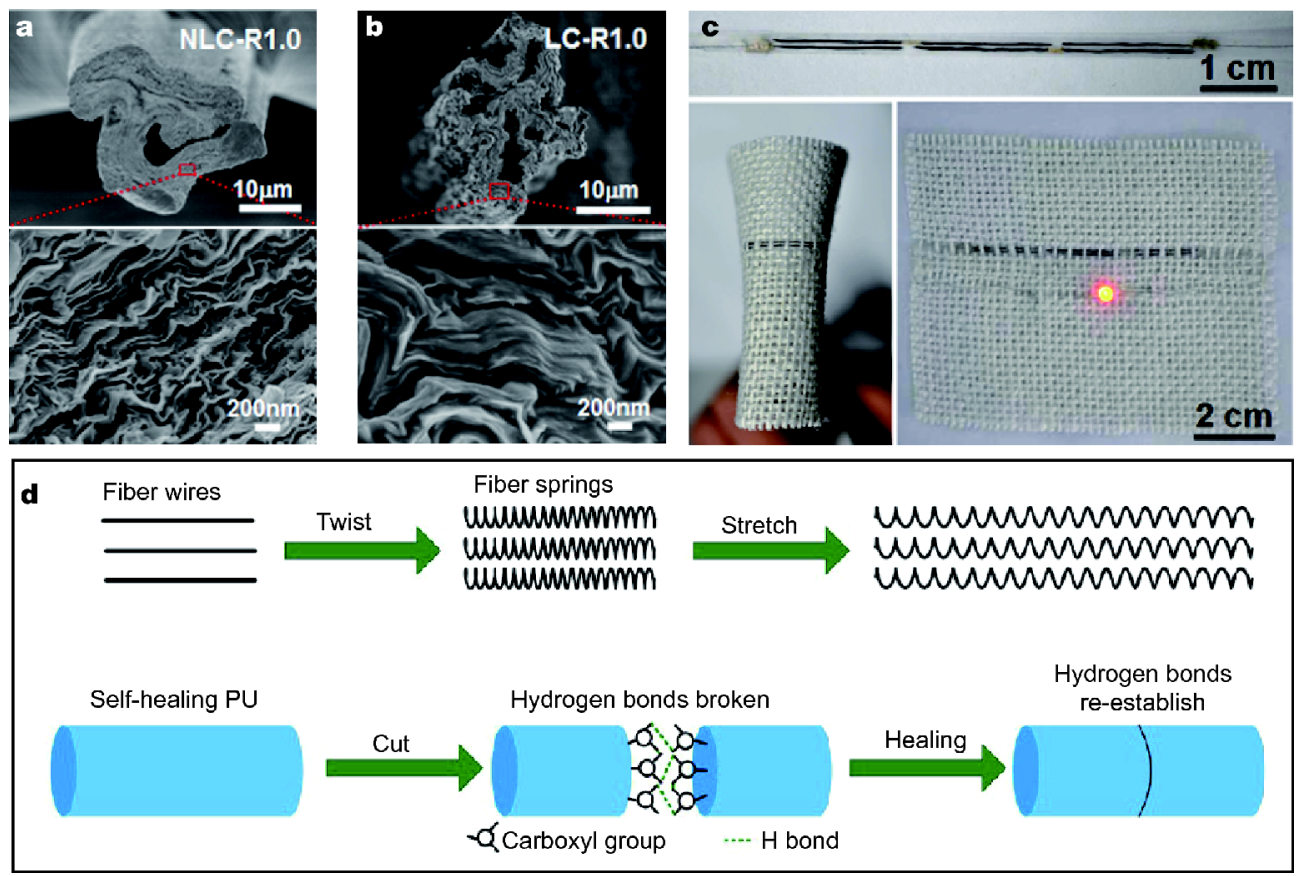

Figure 9 SEM images of the cross-section and the surface of RGO fibers prepared by NLC (a) and LC (b) spinning. R represent the jet stretch ratio. (c) Photo of a $3 \mathrm{~V}$ yarn supercapacitor assembled from six bundles of RGO fibers. This device was sewed into a textile and could power a red lightemission diode (LED) for $5 \mathrm{~min}$ after being charged to $3 \mathrm{~V}$ at a scan rate of $10 \mathrm{mV} \mathrm{s}^{-1}$ for $(5 \mathrm{~min})$. Reproduced with permission from Ref. [89]. Copyright 2015, Elsevier. (d) Schematic diagrams of the stretchable and self-healable mechanism. Reproduced with permission from Ref. [93]. Copyright 2017, the American Chemical Society. 
peline, in which $\mathrm{Fe}_{3} \mathrm{O}_{4}$ nanoparticles act as a catalyst for CNT growth in the subsequent CVD process [90]. The obtained composite fiber has good flexibility and high surface area $\left(79.5 \mathrm{~m}^{2} \mathrm{~g}^{-1}\right)$. When woven into textile electrodes, the composite fibers maintained their flexibility and showed an areal capacitance of $0.98 \mathrm{mF} \mathrm{cm}{ }^{-2}$ and a gravimetric specific capacitance of $200.4 \mathrm{~F} \mathrm{~g}^{-1}$. GO/ MWCNT fibers can also be prepared using a wet-spinning method [91]. After reduction in an acid bath, the composite fiber exhibited enhanced stress strength (385.7 MPa) and better conductivity $\left(210.7 \mathrm{~S} \mathrm{~cm}^{-1}\right)$ compared to pure RGO fibers (193.3 $\mathrm{MPa}$ and $53.3 \mathrm{~S} \mathrm{~cm}^{-1}$ ). This improvement can be explained by the synergistic effect of the interconnection of RGO sheets and partially aligned CNTs. The fiber showed a length capacitance of $351 \mu \mathrm{F} \mathrm{cm}{ }^{-1}$, corresponding to a volumetric capacitance of $38.8 \mathrm{~F} \mathrm{~cm}^{-3}$ at $50 \mathrm{~mA} \mathrm{~cm}^{-3}$.

In addition to modifying the surface structure of graphene fibers, introducing pseudocapacitive materials is a common method for increasing the capacitance of graphene fibers. Manganese dioxide, a typical pseudocapacitive metal oxide, can be deposited on graphene fibers solvothermally [82] and electrochemical deposition [92]. Typically, graphene fiber is submerged in potassium permanganate $\left(\mathrm{KMnO}_{4}\right)$ solution, leading to the uniform deposition of $\mathrm{MnO}_{2}$ particles on the surface of the graphene fiber $\left(\mathrm{GF}-\mathrm{MnO}_{2}\right)$ due to redox reactions between carbon and $\mathrm{KMnO}_{4}$ [82]. The resulting GF- $\mathrm{MnO}_{2}$ composite electrode has an areal capacitance of $42.07 \mathrm{mF} \mathrm{cm}^{-2}$, around 20 times higher than that of the original GF supercapacitor. Based on the aforementioned work [92], Chen et al. deposited $\mathrm{MnO}_{2}$ nanoflowers on core/sheath graphene fibers. This hierarchical structure combines the high EDL capacitance of the graphene network, the good mechanical strength of the graphene fibers, and the pseudocapacitance of $\mathrm{MnO}_{2}$, leading to an increased length capacitance of $143 \mu \mathrm{F} \mathrm{cm}{ }^{-1}$. Recently, a spring-like RGO/MWCNTs/PPy composite fiber has been prepared by incorporating the hydrothermal method with electrodeposition [93]. The outer surface of the fiber was coated with carboxylated polyurethane (PU) which acts as a self-healing material. The composite endows the fiber with several advantages: (1) the MWCNTs increase the specific surface area and the mechanical flexibility; (2) the polypyrrole coating enhances the capacitive performance of the fiber due to its associated redox reactions; (3) the spring-like structure makes the fiber highly stretchable, whereas the interfacial hydrogen bonding of the carboxylated PU allows the fiber supercapacitor to self-heal when little damage occurs (Fig. 9d).
Therefore, the supercapacitor could maintain $82.4 \%$ of its capacitance after being stretched to a strain of $200 \%$. In addition, $54.2 \%$ of its capacitance is maintained after the third self-healing, indicating the good stretchable and self-healing properties of the PPy/RGO/MWCNTs fibers. To promote the application of graphene fibers in flexible supercapacitors, further improvement of their mechanical stability is essential for the maintenance of the structure of the fiber supercapacitors when woven into textiles.

\section{Graphene films}

Graphene films have been used to fabricate flexible supercapacitors over the past few years because of their good flexibility and high surface area. Graphene films can be prepared through various methods including vacuum filtration [94-96], drop-casting [97,98], layer-by-layer assembly [99] and through the compression of graphene aerogel or graphene foam $[100,101]$. However, the electrochemical performance of graphene films is restricted by the restacking of graphene sheets, because agglomeration decreases the specific surface area and results in more difficult diffusion of the electrolyte ions. These issues can be addressed by incorporating additional spacers [94,95] and/or designing highly porous structures. As a typical example, carbon black nanoparticles have been incorporated as spacers and have been used to minimize the restacking of graphene sheets [94]. Through ultrasonication with graphene colloid and subsequent filtration, carbon black particles successfully entered the interspace between the graphene sheets (Fig. 10a, b). The specific capacitance of the pillar graphene electrodes was improved by more than $700 \%$ compared to the original graphene electrodes. CNT is also a commonly used spacer for enhancing the capacitive performance of graphene films. MWCNTs in graphene/MWCNT films increase the basal spacing and bridge the defects for electron transfer between graphene sheets, facilitating the transportation of electrolyte ions and electrons into the inner region of the electrode. The obtained flexible film showed both higher specific capacitance and better rate capability [102]. Pham et al. [103] synthesized a flexible graphene/CNT film via electrostatic interaction between positively charged CNTs and negatively charged graphene oxide sheets. A cationic surfactant (cetyltrimethylammonium bromide: CTAB) was grafted onto the surface of CNTs to generate positively charged CNTs (CTAB-CNTs). After mixing GO and $\mathrm{CTAB}-\mathrm{CNT}$ solutions, a $\mathrm{KOH}$ activation step was used to reduce the GO functional groups and create nanoscale pores in both the SWCNTs and graphene layers. As illustrated in Fig. 10 c, d, graphene layers were pillared 

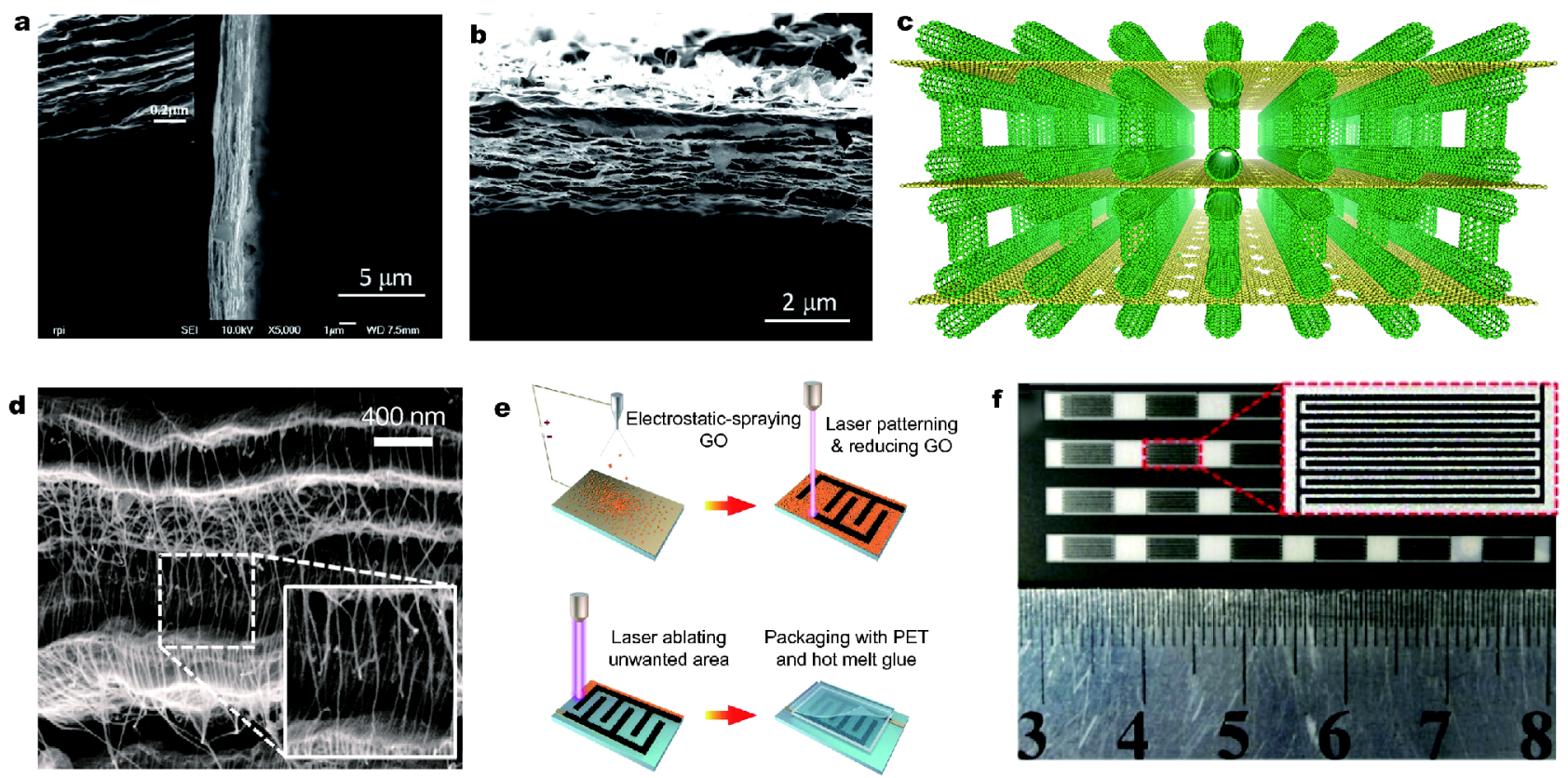

Figure 10 (a) Cross-sectional SEM image of the original GP without CB. (b) Cross sectional HIM images of the pillared GP with the addition of 20 vol.\% CB. Reproduced with permission from Ref. [94]. Copyright 2012, Wiley-VCH. (c) Schematic of the 3D SWCNT-bridged graphene block. KOH activation generates nanoscale pores in the graphene layers. (d) Cross-sectional SEM image containing dangling SWCNTs (magnified in the inset). Reproduced with permission from Ref. [103]. Copyright 2015, the American Chemical Society. (e) Schematic illustration of ultrathin laser-processed graphene based micro-planar supercapacitors. (f) Photographic image of LPG-MPS arrays. (Inset is a single unit). Reproduced with permission from Ref. [106]. Copyright 2016, Elsevier.

by intercalated CNTs, which enhanced the accessible surface area and allowed for fast ion diffusion. When tested in an ionic liquid electrolyte, the supercapacitors fabricated with the as-prepared film delivered high energy and power densities of $110.6 \mathrm{~W} \mathrm{~h} \mathrm{~kg}^{-1}$ and $400 \mathrm{~kW} \mathrm{~kg}^{-1}$, respectively. Microporous graphene films with high specific area can also be generated from freeze-drying solvated graphene [104]. Flexible solid-state supercapacitors based on graphene films prepared by the freeze-drying method exhibited a high specific capacitance of $245 \mathrm{~F} \mathrm{~g}^{-1}$ at $1 \mathrm{~A} \mathrm{~g}^{-1}$ and retained $83 \%$ of their original capacitance after 10,000 cycles. Furthermore, when integrated with a perovskite hybrid solar cell, the supercapacitor can be charged to $0.75 \mathrm{~V}$ and discharged at a current density of $1 \mathrm{~A} \mathrm{~g}^{-1}$ for $45 \mathrm{~s}$. A commercial light scribe CD/DVD optical drive was employed to irradiate a graphene oxide film on a DVD disc [97]. The initially stacked graphene oxide sheets were successfully transformed into exfoliated graphene with a porous structure after laser irradiation. When assembled into a symmetrical supercapacitor, the device exhibited a large areal capacitance of $1.84 \mathrm{mF} \mathrm{cm}^{-2}$ at an extremely large current density of $1000 \mathrm{~A} \mathrm{~g}^{-1}$, indicating good high-rate capability. An electrical resistance change of only $1 \%$ was observed for the laser-scribed graphene after 1000 bending cycles. With the electrodeposition of $\mathrm{MnO}_{2}$ onto the laser scribed graphene, the specific capacitance of resulting microsupercapacitor can be improved to $0.8 \mathrm{~F} \mathrm{~cm}^{-2}$. Moreover, the microsupercapacitor can be directly connected with a solar cell to achieve energy harvesting and storage in a single device [105].

Considering the unique advantages of laser fabrication technology, such as high efficiency, high resolution, and low cost, Xie et al. [106] recently fabricated a microplanar supercapacitor based on laser-processed graphene. A graphene oxide film was first deposited on a PET/Ni substrate by the electrostatic spray deposition. Then, the film was exposed to a laser beam with a specific wavelength to reduce GO. Afterwards, a laser beam with a higher power was used to etch the film for patterning (Fig. 10e). By the use of mature technologies like electrostatic spray deposition, laser treatment, and stencil printing, fine patterning can be achieved on micro-planar supercapacitors (Fig. 10f). On the use of an ionic liquid as the electrolyte, the micro-planar supercapacitor delivers a volumetric energy density of $5.7 \mathrm{~mW} \mathrm{~h} \mathrm{~cm}{ }^{-3}$, which is 3.75 times that of a commercial micro-supercapacitor and 8,785 times that of an aluminum electrolytic capacitor. 

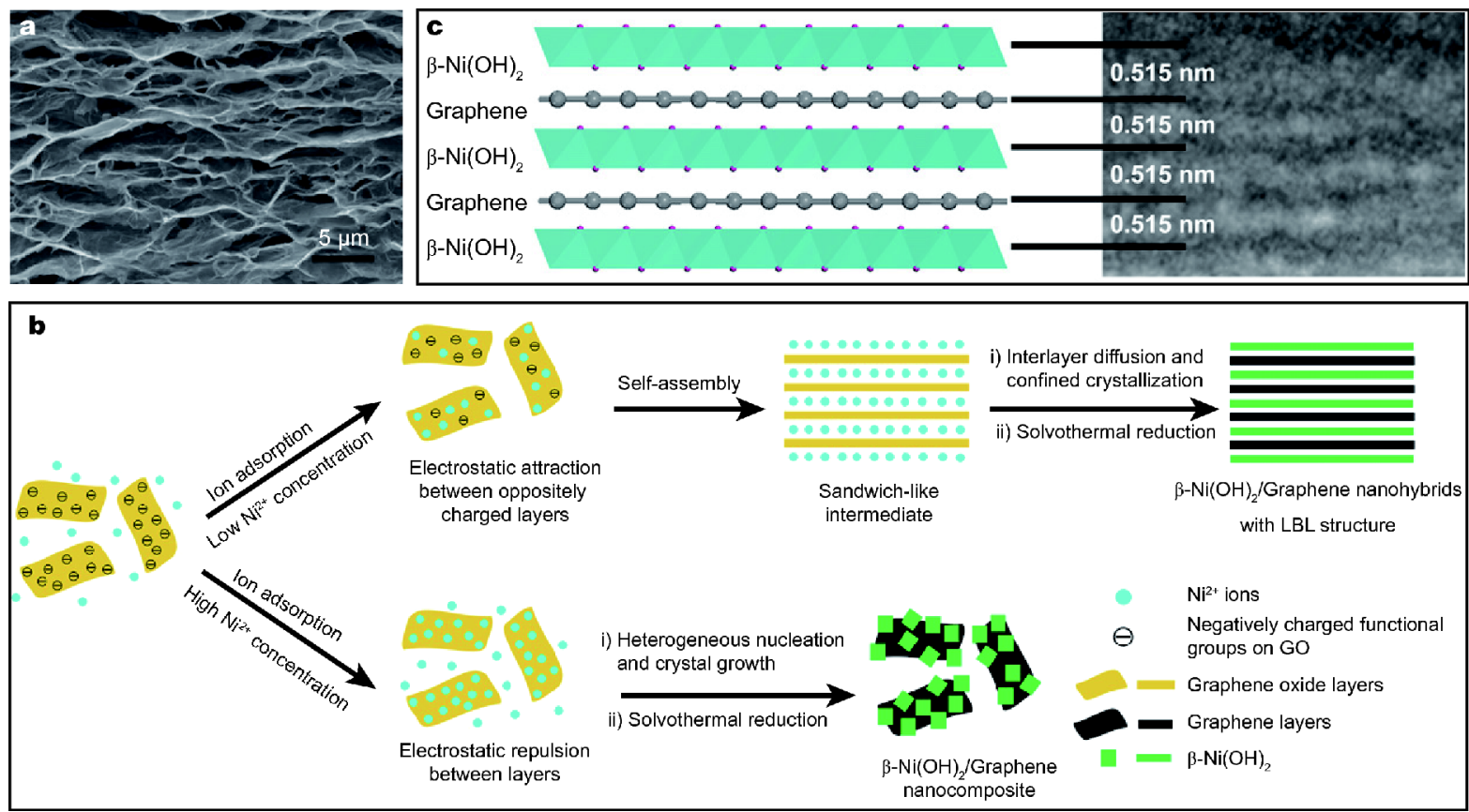

Figure 11 (a) SEM images of the RGO films. Reproduced with permission from Ref. [107]. Copyright 2015, Wiley-VCH. (b) Schematic illustration of the formation mechanism of layer-by-layer b-Ni(OH) $)_{2}$ graphene nanohybrids. (c) HR-TEM image and the structural model of the layer-by-layer nanohybrids. Reproduced with permission from Ref. [108]. Copyright 2012, Elsevier.

This superior performance can be attributed to the planar layout and unique open porous structure of the laserprocessed graphene.

To address the poor processability of aqueous graphene dispersions, a novel strategy has been developed to prepare graphene films from blade-casted graphene hydrogel [107]. Thin GO hydrogel films prepared by blade-casting on glass substrates were pretreated with piranha solution. Then, the reduction of GO using a HI/HAc solution and subsequent freeze-drying enabled the formation of tough RGO films with hierarchically porous structure (Fig. 11a). A flexible supercapacitor assembled with the as-prepared RGO films exhibited a high areal specific capacitance $\left(71 \mathrm{mF} \mathrm{cm}^{-2}\right)$ and excellent rate performance $(79 \%$ capacitance retention at a discharge rate of $\left.100 \mathrm{~mA} \mathrm{~cm}^{-2}\right)$. This capacitive performance can be attributed to the advantages of the 3D macroporous/mesoporous structure, such as superior toughness, large specific area, and good electrical conductivity.

The combination of graphene films with pseudocapacitive materials is also an effective strategy to enhance the capacitive performance of graphene-based supercapacitors. The in-situ electrochemical polymerization of polyaniline nanorods on RGO paper allowed for the preparation of a flexible graphene-polyaniline composite paper [98]. The composite electrodes exhibited an enhanced capacitance of $763 \mathrm{~F} \mathrm{~g}^{-1}$ and better cycling stability compared to pure RGO paper and pure polyaniline electrodes. An all-solid-state thin-film supercapacitor with transition metal hydroxides exhibited a high volume capacitance of $660.8 \mathrm{~F} \mathrm{~cm}^{-3}$ with negligible degradation after 2,000 cycles [108]. As shown in Fig. 11b, graphene sheets with different electrostatic charges self-assemble into sandwich-like intermediates by adjusting the concentration of $\mathrm{Ni}^{2+}$ ions in the precursor solution. The layer-by-layer structure of the $\beta-\mathrm{Ni}(\mathrm{OH})_{2} /$ graphene nanohybrids (Fig. 11c) was finally obtained via solvothermal reduction. The ultrathin layer-by-layer structure provides superior flexibility and maximizes the utilization of the active materials. The graphene layers between $\beta-\mathrm{Ni}(\mathrm{OH})_{2}$ are beneficial for facilitating fast electron transfer, thus increasing the rate performance. In addition to combining the graphene film with pseudocapacitive materials at the nanoscale, direct doping of heteroatoms into carbon scaffolds also increases the pseudocapacitance [109-111]. It has been shown that $\mathrm{C}-\mathrm{S}$ bonds near edges or defects are active sites which influence the charging of the electrical double layer and facilitate the pseudocapacitive behavior [112]. Therefore, a bottom-up method has been developed to synthesize sulfur doped graphene (SG) films 

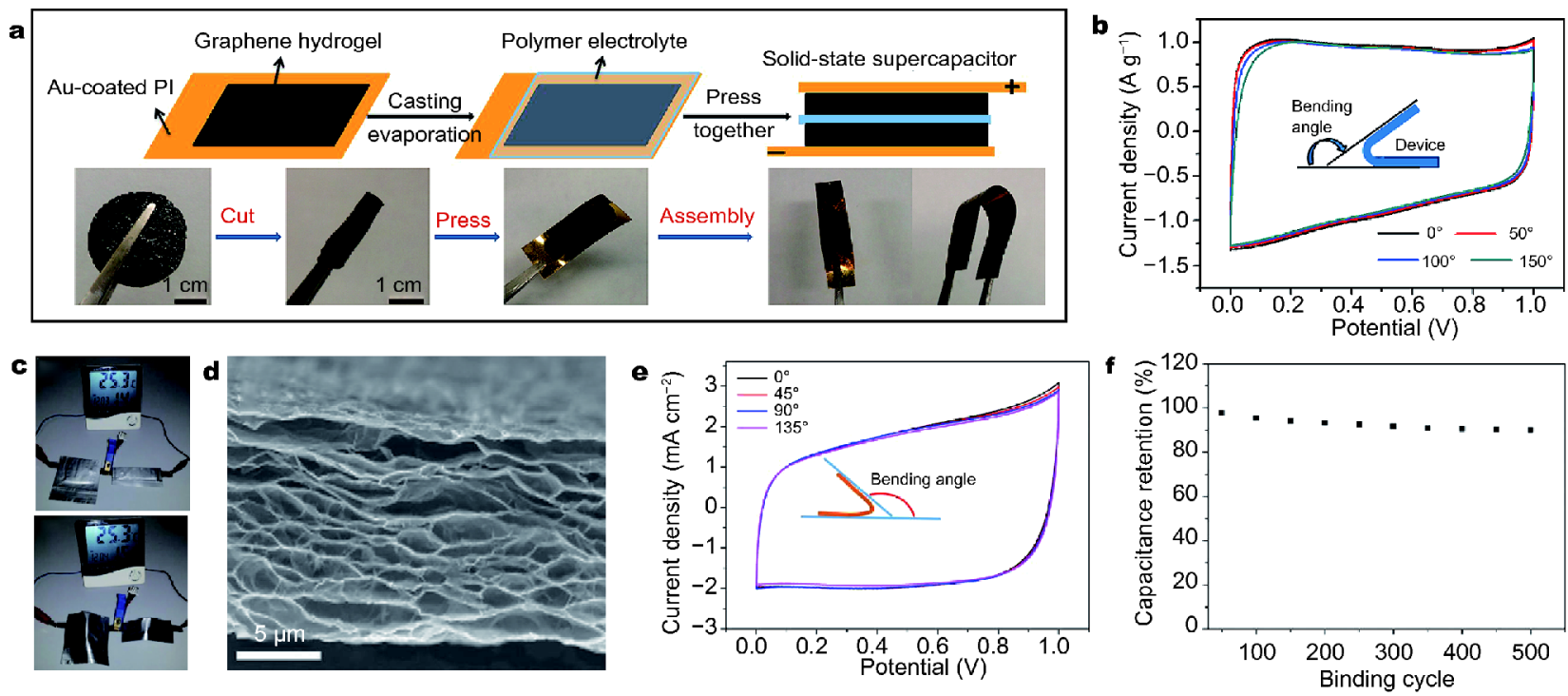

Figure 12 (a) Schematic diagram and photographs of the fabrication process of flexible solid-state supercapacitors based on graphene hydrogel films. (b) CV curves of the flexible solid-state device at $10 \mathrm{mV} \mathrm{s}^{-1}$ for different bending angles. Reproduced with permission from Ref. [115]. Copyright 2013, the American Chemical Society. (c) Two devices connected in series can power the digital temperature and humidity meter at both a normal (right) and bending (left) state. Reproduced with permission from Ref. [116]. Copyright 2014, the Royal Society of Chemistry. (d) Cross-section SEM image of a 3D porous RGO film after long-term reduction. (e) CV curves of an all solid-state supercapacitor tested at a scan rate of $50 \mathrm{mV} \mathrm{s}$ under different bending angles. (f) The device retains about $91 \%$ of the initial capacitance after 500 cycles when tested under the bent state. Reproduced with permission from Ref. [117]. Copyright 2016, Wiley-VCH.

in micro-supercapacitors [109]. Typically, peripherical trisulfur-annulated hexa-peri-hexabenzocoronene (SHBC) dispersion is coated on a silicon wafer. After covered with a thin Au protecting layer, the SHBC film is thermally transformed into the SG film. The film showed a high volumetric capacitance of $582 \mathrm{~F} \mathrm{~cm}^{-3}$ at a scan rate of $10 \mathrm{mV} \mathrm{s}^{-1}$ and maintains a volumetric capacitance of $8.1 \mathrm{~F} \mathrm{~cm}^{-3}$ at an ultrahigh scan rate of $2000 \mathrm{~V} \mathrm{~s}^{-1}$, indicating its excellent rate stability.

\section{D graphene framework}

Because of their single fiber or ultrathin structures, graphene fibers and graphene films exhibit good electrochemical performance. However, the challenges of knitting fiber supercapacitors into textiles or increasing mass loading of film supercapacitors without capacitance degradation remain to be overcome. To optimize the structure of graphene active materials for further improving performance, 3D porous graphene frameworks, also called graphene foam, have been developed as supercapacitor electrodes recently [113-115]. Flexible 3D graphene frameworks have high electrical conductivity and good wettability, facilitating the fast diffusion of electrolyte ions and contributing to a high power density. In addition, due to the interconnected and macroporous structure, high mass-loading of active materials can be realized in the 3D graphene framework without sacrificing capacitance. Xu et al. [115] reported a flexible solidstate supercapacitor based on RGO hydrogel synthesized by a hydrothermal reduction method (Fig. 12a). The prepared RGO hydrogel with a porous structure was pressed on a gold-coated polyimide current collector. The obtained supercapacitor exhibited an areal specific capacitance of $372 \mathrm{mF} \mathrm{cm}^{-2}$ and a gravimetric specific capacitance of $186 \mathrm{~F} \mathrm{~g}^{-1}$ and the CV curves showed only a few differences with different bending states (Fig. 12b). A milder hydrothermal method performed at a relatively low temperature was used to synthesize ordered 3D graphene [116]. Flexible binder-free supercapacitors were fabricated by pressing the as-synthesized graphene onto a nickel substrate and the resulting device exhibited a gravimetric capacitance of $220 \mathrm{~F} \mathrm{~g}^{-1}$. Two of these supercapacitor devices connected in series were able to power a digital thermal-meter for $15 \mathrm{~min}$ while using a soft aluminum packaging film as an encapsulating material (Fig. 12c). Recently, a two-step method was used to synthesize 3D hierarchical porous graphene [117]. The filtration assembly of partially reduced GO followed by subsequent freeze-casting led to the formation of cellular graphene. Freeze-casting is a versatile, readily accessible 
and inexpensive solution-phase technique that uses the controlled crystallization of a suspension to induce ordered hierarchical porous architectures [118]. However, directly freeze-cast GO dispersions cannot adopt an ordered structure, and instead form a randomly oriented porous brittle monolith. Therefore, pre-reduction with a carefully controlled reduction time is crucial to obtain appropriate "particles", which are the source of the continuous 3D porous network. Upon optimization of the fabrication conditions, the obtained graphene foam with a highly porous structure (Fig. 12d) exhibited superior electrical conductivity and good mechanical strength. When used as supercapacitor electrodes, only $9 \%$ capacitance decay was observed after $500 \mathrm{CV}$ cycles and bending tests $\left(0^{\circ}\right.$ to $135^{\circ}$, Fig. 12e, f).

The pseudocapacitive performance can be further improved throng incorporating 3D graphene framework. Yu et al. [119] synthesized RGO foam by a dipping and dry method with $\mathrm{Ni}$ foam as a template. Polyaniline nanowire arrays were polymerized in-situ on the surface of the RGO facilitated by the $\pi-\pi$ interaction between polyaniline and RGO. The uniform deposition reduced the diffusion length of ions in the PANI phase during rapid redox reactions and ensured high utilization of the electrode materials. The synergetic effect of PANI and the porous RGO foam caused the energy and power densities of the composite electrode to be approximately 8 times higher than those of the pure RGO foam electrodes. Owing to the low-cost, high specific surface area, and regularly interconnected pore channels, ordered mesoporous carbon (OMC) is a promising material for electric double layer capacitors [120-122]. Zhi et al. [123] combined CVD-grown graphene foam, OMC, and Ag nanowires to form a 3D interconnected network, named AgGF-OMC. The graphene foam acts as a flexible and conductive scaffold while the Ag nanowires act as conductive additives. This hierarchical structure exhibited an ultrahigh electrical conductivity of $741 \mathrm{~S} \mathrm{~cm}^{-1}$ and an amplified specific capacitance of $213 \mathrm{~F} \mathrm{~g}^{-1}$ compared to the original graphene foam $\left(39 \mathrm{~F} \mathrm{~g}^{-1}\right)$. Surface modification with heteroatom functional groups on the carbon scaffold is another strategy to increase the pseudocapacitance of the carbon materials. Qin et al. [124] used melamine foam as a template to fabricate a flexible $\mathrm{O} / \mathrm{N}$ enriched graphene foam. During the preparation process, a compression/hot curing strategy was used to prevent the graphene from shrinking and to simultaneously introduce high concentrations of $\mathrm{O} / \mathrm{N}$ functional groups. The obtained graphene foam exhibited good robustness as determined from bending tests. No obvious resistance change was observed when the material was bent to $180^{\circ}$ after 500 cycles. After a compacting step, the graphene foam exhibited a volumetric capacitance of $106 \mathrm{~F} \mathrm{~cm}^{-3}$ at a current density of $1 \mathrm{~A} \mathrm{~g} \mathrm{~g}^{-1}$. It was determined that the $\mathrm{N} /$ O functional groups were responsible for approximately $65 \%$ of the total capacitance, indicating the successful doping of pseudocapacitive functional groups on the carbon surface.

\section{SUMMARY AND OUTLOOK}

Great progress has been made in the development of flexible supercapacitors based on CNTs and graphene materials. Recent advances in the fabrication of CNT nanostructures and graphene for fabricating flexible supercapacitors have been summarized in this review. There is no doubt that the nanostructures of CNTs and graphene influence the capacitive performance and determine the mechanical properties of the resulting supercapacitors to a large extent. The nanostructures based on CNT and graphene can be divided into three major types: (1) 1D fibers or yarns, (2) 2D films and (3) 3D foams or arrays, each with their own advantages and disadvantages. Fibers and films usually possess excellent mechanical properties, and extremely high specific capacitance can be achieved with $1 \mathrm{D}$ or $2 \mathrm{D}$ nanostructures. However, the excellent electrochemical performance is often highly dependent on the choice of appropriate substrates or a relatively low mass loading. 3D nanostructures result in materials with large surface areas and good contact with the electrolyte, which leads to better electrochemical performance under high mass loading conditions. However, the mechanical properties of 3D structures are not always as good as the $1 \mathrm{D}$ or $2 \mathrm{D}$ structures. Based on the above discussion, it is clear that $\mathrm{CNTs}$ and graphene are promising materials for constructing flexible supercapacitors, but many challenges remain. When high performance flexible supercapacitors are constructed, the following issues still need to be addressed:

(1) It is essential to complement the structural design of the CNTs and graphene depending on the specific functions desired. For instance, 3D foams usually have a large accessible ion surface, but have poor mechanical strength compared to 1D fibers. However, 1D fibers are usually not as compressible as 3D foams.

(2) Flexible supercapacitors have been fabricated with the recent advances in structural design and novel strategies for modulation of the properties of CNTs and graphene. However, efforts are still needed to further improve the flexibility of these materials to meet the re- 
quirements of practical application. The combination of intrinsic electrode materials and novel device configuration is a promising strategy to achieve this goal.

(3) It is highly desirable to develop standardized testing procedures and comprehensive evaluation standards for flexible supercapacitors. More detailed mechanistic statistics instead of the simple electrochemical curves obtained under deformable states would allow for the proper evaluation of the real flexibility of supercapacitors and assessment of their potential application.

(4) Low cost and scalable production is important for practical applications. To reduce the cost of flexible and wearable electronics, large-scale synthetic methods and abundant and cheap raw materials would be beneficial. Mature technologies such as printing and laser irradiation have shown promise for the economical fabrication of flexible electrodes.

(5) Integration of flexible supercapacitors with other devices is a promising direction for future research. For example, the combination of flexible supercapacitors with energy harvesting devices such as solar cells may be able to achieve self-powering devices. These integrated devices combining different functions show great potential in novel portable and wearable electronics.

\section{Received 28 August 2017; accepted 31 October 2017; published online 15 December 2017}

1 Liu W, Song MS, Kong B, et al. Flexible and stretchable energy storage: recent advances and future perspectives. Adv Mater, 2017, 29: 1603436

2 Wen L, Li F, Cheng HM. Carbon nanotubes and graphene for flexible electrochemical energy storage: from materials to devices. Adv Mater, 2016, 28: 4306-4337

3 Zhang J, Zhao XS. On the configuration of supercapacitors for maximizing electrochemical performance. ChemSusChem, 2012, 5: 818-841

4 Yu M, Wang Z, Han Y, et al. Recent progress in the development of anodes for asymmetric supercapacitors. J Mater Chem A, 2016, 4: $4634-4658$

5 Ge J, Lan M, Liu W, et al. Graphene quantum dots as efficient, metal-free, visible-light-active photocatalysts. Sci China Mater, 2016, 59: 12-19

$6 \mathrm{Lu} \mathrm{K}, \mathrm{Hu} \mathrm{Z}$, Xiang Z, et al. Cation intercalation in manganese oxide nanosheets: effects on lithium and sodium storage. Angew Chem, 2016, 128: 10604-10608

7 Huang Q, Wang D, Zheng Z. Textile-based electrochemical energy storage devices. Adv Energ Mater, 2016, 6: 1600783

8 Liu L, Niu Z, Chen J. Unconventional supercapacitors from nanocarbon-based electrode materials to device configurations. Chem Soc Rev, 2016, 45: 4340-4363

9 Guo K, Yu N, Hou Z, et al. Smart supercapacitors with deformable and healable functions. J Mater Chem A, 2017, 5: 16-30

10 Gelinck GH, Huitema HEA, van Veenendaal E, et al. Flexible active-matrix displays and shift registers based on solution-pro- cessed organic transistors. Nat Mater, 2004, 3: 106-110

11 Moonen PF, Yakimets I, Huskens J. Fabrication of transistors on flexible substrates: from mass-printing to high-resolution alternative lithography strategies. Adv Mater, 2012, 24: 5526-5541

12 Tee BCK, Wang C, Allen R, et al. An electrically and mechanically self-healing composite with pressure- and flexion-sensitive properties for electronic skin applications. Nat Nanotech, 2012, 7: 825-832

13 Hammock ML, Chortos A, Tee BCK, et al. 25th Anniversary Article: the evolution of electronic skin (e-skin): a brief history, design considerations, and recent progress. Adv Mater, 2013, 25: 5997-6038

14 Zhang X, Zhang H, Lin Z, et al. Recent advances and challenges of stretchable supercapacitors based on carbon materials. Sci China Mater, 2016, 59: 475-494

15 Fan Z, Yan J, Zhi L, et al. A three-dimensional carbon nanotube/ graphene sandwich and its application as electrode in supercapacitors. Adv Mater, 2010, 22: 3723-3728

16 Qin J, Zhou F, Xiao H, et al. Mesoporous polypyrrole-based graphene nanosheets anchoring redox polyoxometalate for allsolid-state micro-supercapacitors with enhanced volumetric capacitance. Sci China Mater, 2017, doi: 10.1007/s40843-017-9132-8

17 Li YX, Gong ZL, Yang Y. Synthesis and characterization of $\mathrm{Li}_{2}$ $\mathrm{MnSiO}_{4} / \mathrm{C}$ nanocomposite cathode material for lithium ion batteries. J Power Sources, 2007, 174: 528-532

18 Cao AM, Hu JS, Liang HP, et al. Self-assembled vanadium pentoxide $\left(\mathrm{V}_{2} \mathrm{O}_{5}\right)$ hollow microspheres from nanorods and their application in lithium-ion batteries. Angew Chem Int Ed, 2005, 44: 4391-4395

19 Cai X, Zhang C, Zhang S, et al. Application of carbon fibers to flexible, miniaturized wire/fiber-shaped energy conversion and storage devices. J Mater Chem A, 2017, 5: 2444-2459

$20 \mathrm{Wu} \mathrm{Z}$, Zhang X. N,O-codoped porous carbon nanosheets for capacitors with ultra-high capacitance. Sci China Mater, 2016, 59: 547-557

21 Wu S, Zhu Y. Highly densified carbon electrode materials towards practical supercapacitor devices. Sci China Mater, 2017, 60: 25-38

22 Lu K, Zhang J, Wang Y, et al. Interfacial deposition of threedimensional nickel hydroxide nanosheet-graphene aerogel on $\mathrm{Ni}$ wire for flexible fiber asymmetric supercapacitors. ACS Sustain Chem Eng, 2017, 5: 821-827

23 Yin H, Tang Z. Ultrathin two-dimensional layered metal hydroxides: an emerging platform for advanced catalysis, energy conversion and storage. Chem Soc Rev, 2016, 45: 4873-4891

24 Mendoza-Sánchez B, Gogotsi Y. Synthesis of two-dimensional materials for capacitive energy storage. Adv Mater, 2016, 28: 6104-6135

25 Gao Y. Graphene and polymer composites for supercapacitor applications: a review. Nanoscale Res Lett, 2017, 12: 387

26 Wang K, Zhang X, Sun X, et al. Conducting polymer hydrogel materials for high-performance flexible solid-state supercapacitors. Sci China Mater, 2016, 59: 412-420

27 Wang $\mathrm{K}, \mathrm{Wu} \mathrm{H}$, Meng $\mathrm{Y}$, et al. Conducting polymer nanowire arrays for high performance supercapacitors. Small, 2014, 10: 1431

28 Zhang G, Jin X, Li H, et al. N-doped crumpled graphene: bottomup synthesis and its superior oxygen reduction performance. Sci China Mater, 2016, 59: 337-347

29 Shao Y, El-Kady MF, Wang LJ, et al. Graphene-based materials 
for flexible supercapacitors. Chem Soc Rev, 2015, 44: 3639-3665

30 Zhang J, Dai L. Nitrogen, phosphorus, and fluorine tri-doped graphene as a multifunctional catalyst for self-powered electrochemical water splitting. Angew Chem Int Ed, 2016, 55: 1329613300

$31 \mathrm{Yu}$ M, Zhou S, Liu Y, et al. Long life rechargeable $\mathrm{Li}_{-} \mathrm{O}_{2}$ batteries enabled by enhanced charge transfer in nanocable-like Fe@Ndoped carbon nanotube catalyst. Sci China Mater, 2017, 60: 415426

32 Ma Z, Tao L, Liu D, et al. Ultrafine nano-sulfur particles anchored on in situ exfoliated graphene for lithium-sulfur batteries. J Mater Chem A, 2017, 5: 9412-9417

33 Liu Z, Zhao Z, Wang Y, et al. In situ exfoliated, edge-rich, oxygen-functionalized graphene from carbon fibers for oxygen electrocatalysis. Adv Mater, 2017, 29: 1606207

34 Wang S, Jiang SP. Prospects of fuel cell technologies. Nat Sci Rev, 2017, nww099

35 Yan D, Li Y, Huo J, et al. Defect chemistry of nonprecious-metal electrocatalysts for oxygen reactions. Adv Mater, 2017, 414: 1606459

36 Dong L, Xu C, Li Y, et al. Flexible electrodes and supercapacitors for wearable energy storage: a review by category. J Mater Chem A, 2016, 4: 4659-4685

37 Iijima S. Helical microtubules of graphitic carbon. Nature, 1991, 354: $56-58$

38 De Volder MFL, Tawfick SH, Baughman RH, et al. Carbon nanotubes: present and future commercial applications. Science, 2013, 339: 535-539

39 Park S, Vosguerichian M, Bao Z. A review of fabrication and applications of carbon nanotube film-based flexible electronics. Nanoscale, 2013, 5: 1727-1752

40 Yang F, Wang X, Zhang D, et al. Chirality-specific growth of single-walled carbon nanotubes on solid alloy catalysts. Nature, 2014, 510: 522-524

41 Niu Z, Luan P, Shao Q, et al. A "skeleton/skin" strategy for preparing ultrathin free-standing single-walled carbon nanotube/ polyaniline films for high performance supercapacitor electrodes. Energ Environ Sci, 2012, 5: 8726-8733

42 Kang YJ, Chung $\mathrm{H}$, Han $\mathrm{CH}$, et al. All-solid-state flexible supercapacitors based on papers coated with carbon nanotubes and ionic-liquid-based gel electrolytes. Nanotechnology, 2012, 23: 289501

43 Niu Z, Dong H, Zhu B, et al. Highly stretchable, integrated supercapacitors based on single-walled carbon nanotube films with continuous reticulate architecture. Adv Mater, 2013, 25: 10581064

44 Zeng S, Chen H, Cai F, et al. Electrochemical fabrication of carbon nanotube/polyaniline hydrogel film for all-solid-state flexible supercapacitor with high areal capacitance. J Mater Chem A, 2015, 3: 23864-23870

$45 \mathrm{Yu}$ J, Lu W, Pei S, et al. Omnidirectionally stretchable highperformance supercapacitor based on isotropic buckled carbon nanotube films. ACS Nano, 2016, 10: 5204-5211

46 Lv T, Yao Y, Li N, et al. Highly stretchable supercapacitors based on aligned carbon nanotube/molybdenum disulfide composites. Angew Chem Int Ed, 2016, 55: 9191-9195

47 Li J, Lu W, Yan Y, et al. High performance solid-state flexible supercapacitor based on $\mathrm{Fe}_{3} \mathrm{O}_{4} /$ carbon nanotube/polyaniline ternary films. J Mater Chem A, 2017, 5: 11271-11277
Zhao J, Chen J, Xu S, et al. Hierarchical nimn layered double hydroxide/carbon nanotubes architecture with superb energy density for flexible supercapacitors. Adv Funct Mater, 2014, 24: 2938-2946

49 Zhao MQ, Ren CE, Ling Z, et al. Flexible Mxene/carbon nanotube composite paper with high volumetric capacitance. Adv Mater, 2015, 27: 339-345

50 Yuksel R, Sarioba Z, Cirpan A, et al. Transparent and flexible supercapacitors with single walled carbon nanotube thin film electrodes. ACS Appl Mater Interfaces, 2014, 6: 15434-15439

51 Du L, Yang P, Yu X, et al. Flexible supercapacitors based on carbon nanotube $/ \mathrm{MnO}_{2}$ nanotube hybrid porous films for wearable electronic devices. J Mater Chem A, 2014, 2: 17561-17567

52 Chen Y, Du L, Yang P, et al. Significantly enhanced robustness and electrochemical performance of flexible carbon nanotubebased supercapacitors by electrodepositing polypyrrole. J Power Sources, 2015, 287: 68-74

53 Yu M, Zhang Y, Zeng Y, et al. Water surface assisted synthesis of large-scale carbon nanotube film for high-performance and stretchable supercapacitors. Adv Mater, 2014, 26: 4724-4729

54 de Souza VHR, Oliveira MM, Zarbin AJG. Thin and flexible allsolid supercapacitor prepared from novel single wall carbon nanotubes/polyaniline thin films obtained in liquid-liquid interfaces. J Power Sources, 2014, 260: 34-42

55 Shin SR, Farzad R, Tamayol A, et al. A bioactive carbon nanotube-based ink for printing $2 \mathrm{D}$ and $3 \mathrm{D}$ flexible electronics. Adv Mater, 2016, 28: 3280-3289

56 Song L, Cao X, Li L, et al. General method for large-area films of carbon nanomaterials and application of a self-assembled carbon nanotube film as a high-performance electrode material for an all-solid-state supercapacitor. Adv Funct Mater, 2017, 27: 1700474

57 Chen C, Cao J, Lu Q, et al. Foldable all-solid-state supercapacitors integrated with photodetectors. Adv Funct Mater, 2017, 27: 1604639

58 Lee H, Kim H, Cho MS, et al. Fabrication of polypyrrole (PPy)/ carbon nanotube (CNT) composite electrode on ceramic fabric for supercapacitor applications. Electrochim Acta, 2011, 56: 7460-7466

59 Lu X, Dou H, Yuan C, et al. Polypyrrole/carbon nanotube nanocomposite enhanced the electrochemical capacitance of flexible graphene film for supercapacitors. J Power Sources, 2012, 197: 319-324

60 Huang F, Vanhaecke E, Chen D. In situ polymerization and characterizations of polyaniline on MWCNT powders and aligned MWCNT films. Catal Today, 2010, 150: 71-76

61 Zhang H, Cao G, Yang Y. Carbon nanotube arrays and their composites for electrochemical capacitors and lithium-ion batteries. Energ Environ Sci, 2009, 2: 932-943

62 Cai Z, Li L, Ren J, et al. Flexible, weavable and efficient microsupercapacitor wires based on polyaniline composite fibers incorporated with aligned carbon nanotubes. J Mater Chem A, 2013, 1: 258-261

$63 \mathrm{Yu} \mathrm{D}$, Goh K, Wang H, et al. Scalable synthesis of hierarchically structured carbon nanotube-graphene fibres for capacitive energy storage. Nat Nanotech, 2014, 9: 555-562

64 Huang F, Lou F, Chen D. Exploring aligned-carbon-nanotubes@polyaniline arrays on household $\mathrm{Al}$ as supercapacitors. ChemSusChem, 2012, 5: 888-895

65 Lin H, Li L, Ren J, et al. Conducting polymer composite film incorporated with aligned carbon nanotubes for transparent, 
flexible and efficient supercapacitor. Sci Rep, 2013, 3: 1353

66 Chen T, Peng H, Durstock M, et al. High-performance transparent and stretchable all-solid supercapacitors based on highly aligned carbon nanotube sheets. Sci Rep, 2014, 4: 3612

67 Pan S, Lin H, Deng J, et al. Novel wearable energy devices based on aligned carbon nanotube fiber textiles. Adv Energ Mater, 2015, 5: 1401438

68 Malik R, Zhang L, McConnell C, et al. Three-dimensional, freestanding polyaniline/carbon nanotube composite-based electrode for high-performance supercapacitors. Carbon, 2017, 116: 579590

69 Cherusseri J, Kar KK. Ultra-flexible fibrous supercapacitors with carbon nanotube/polypyrrole brush-like electrodes. J Mater Chem A, 2016, 4: 9910-9922

70 Zhang G, Song Y, Zhang H, et al. Radially aligned porous carbon nanotube arrays on carbon fibers: a hierarchical $3 \mathrm{D}$ carbon nanostructure for high-performance capacitive energy storage. Adv Funct Mater, 2016, 26: 3012-3020

71 Reit R, Nguyen J, Ready W. Growth time performance dependence of vertically aligned carbon nanotube supercapacitors grown on aluminum substrates. Electrochim Acta, 2013, 91: 96100

72 Zhao W, Li Y, Wu S, et al. Highly stable carbon nanotube/ polyaniline porous network for multifunctional applications. ACS Appl Mater Interfaces, 2016, 8: 34027-34033

73 Li P, Kong C, Shang Y, et al. Highly deformation-tolerant carbon nanotube sponges as supercapacitor electrodes. Nanoscale, 2013, 5: $8472-8479$

74 Cheng X, Gui X, Lin Z, et al. Three-dimensional $\alpha-\mathrm{Fe}_{2} \mathrm{O}_{3} /$ carbon nanotube sponges as flexible supercapacitor electrodes. J Mater Chem A, 2015, 3: 20927-20934

75 Li P, Shi E, Yang Y, et al. Carbon nanotube-polypyrrole core-shell sponge and its application as highly compressible supercapacitor electrode. Nano Res, 2013, 7: 209-218

76 Wang K, Meng Q, Zhang Y, et al. High-performance two-ply yarn supercapacitors based on carbon nanotubes and polyaniline nanowire arrays. Adv Mater, 2013, 25: 1494-1498

77 Zhang D, Miao M, Niu H, et al. Core-spun carbon nanotube yarn supercapacitors for wearable electronic textiles. ACS Nano, 2014, 8: 4571-4579

78 Shang Y, Wang C, He X, et al. Self-stretchable, helical carbon nanotube yarn supercapacitors with stable performance under extreme deformation conditions. Nano Energ, 2015, 12: 401-409

79 Li P, Yang Y, Shi E, et al. Core-double-shell, carbon nanotube@polypyrrole@ $\mathrm{MnO}_{2}$ sponge as freestanding, compressible supercapacitor electrode. ACS Appl Mater Interfaces, 2014, 6: 5228-5234

80 Dong $\mathrm{Z}$, Jiang $\mathrm{C}$, Cheng $\mathrm{H}$, et al. Facile fabrication of light, flexible and multifunctional graphene fibers. Adv Mater, 2012, 24: 1856-1861

81 Cong HP, Ren XC, Wang P, et al. Wet-spinning assembly of continuous, neat and macroscopic graphene fibers. Sci Rep, 2012, 2: 613

82 Li X, Zhao T, Chen Q, et al. Flexible all solid-state supercapacitors based on chemical vapor deposition derived graphene fibers. Phys Chem Chem Phys, 2013, 15: 17752-17757

83 Li X, Zhao T, Wang K, et al. Directly drawing self-assembled, porous, and monolithic graphene fiber from chemical vapor deposition grown graphene film and its electrochemical properties. Langmuir, 2011, 27: 12164-12171
84 Meng Y, Zhao Y, Hu C, et al. All-graphene core-sheath microfibers for all-solid-state, stretchable fibriform supercapacitors and wearable electronic textiles. Adv Mater, 2013, 25: 2326-2331

85 Li Y, Sheng K, Yuan W, et al. A high-performance flexible fibreshaped electrochemical capacitor based on electrochemically reduced graphene oxide. Chem Commun, 2013, 49: 291-293

$86 \mathrm{Hu} \mathrm{Y}$, Cheng $\mathrm{H}$, Zhao F, et al. All-in-one graphene fiber supercapacitor. Nanoscale, 2014, 6: 6448-6451

87 McDonough JR, Choi JW, Yang Y, et al. Carbon nanofiber supercapacitors with large areal capacitances. Appl Phys Lett, 2009, 95: 243109

$88 \mathrm{Kim} \mathrm{JH}$, Kang SH, Zhu K, et al. Ni-NiO core-shell inverse opal electrodes for supercapacitors. Chem Commun, 2011, 47: 52145216

89 Chen S, Ma W, Cheng Y, et al. Scalable non-liquid-crystal spinning of locally aligned graphene fibers for high-performance wearable supercapacitors. Nano Energ, 2015, 15: 642-653

90 Cheng H, Dong Z, Hu C, et al. Textile electrodes woven by carbon nanotube-graphene hybrid fibers for flexible electrochemical capacitors. Nanoscale, 2013, 5: 3428-3434

91 Ma Y, Li P, Sedloff JW, et al. Conductive graphene fibers for wireshaped supercapacitors strengthened by unfunctionalized fewwalled carbon nanotubes. ACS Nano, 2015, 9: 1352-1359

92 Chen $\mathrm{Q}$, Meng $\mathrm{Y}, \mathrm{Hu} \mathrm{C}$, et al. $\mathrm{MnO}_{2}$-modified hierarchical graphene fiber electrochemical supercapacitor. J Power Sources, 2014, 247: 32-39

93 Wang S, Liu N, Su J, et al. Highly stretchable and self-healable supercapacitor with reduced graphene oxide based fiber springs. ACS Nano, 2017, 11: 2066-2074

94 Wang G, Sun X, Lu F, et al. Flexible pillared graphene-paper electrodes for high-performance electrochemical supercapacitors. Small, 2012, 8: 452-459

95 Yang X, Zhu J, Qiu L, et al. Bio-inspired effective prevention of restacking in multilayered graphene films: towards the next generation of high-performance supercapacitors. Adv Mater, 2011, 23: 2833-2838

96 Cheng Y, Lu S, Zhang H, et al. Synergistic effects from graphene and carbon nanotubes enable flexible and robust electrodes for high-performance supercapacitors. Nano Lett, 2012, 12: 42064211

97 El-Kady MF, Strong V, Dubin S, et al. Laser scribing of highperformance and flexible graphene-based electrochemical capacitors. Science, 2012, 335: 1326-1330

98 Cong HP, Ren XC, Wang P, et al. Flexible graphene-polyaniline composite paper for high-performance supercapacitor. Energ Environ Sci, 2013, 6: 1185

99 Yoo JJ, Balakrishnan K, Huang J, et al. Ultrathin planar graphene supercapacitors. Nano Lett, 2011, 11: 1423-1427

100 Liu F, Song S, Xue D, et al. Folded structured graphene paper for high performance electrode materials. Adv Mater, 2012, 24: 1089-1094

101 Li N, Lv T, Yao Y, et al. Compact graphene/ $\mathrm{MoS}_{2}$ composite films for highly flexible and stretchable all-solid-state supercapacitors. J Mater Chem A, 2017, 5: 3267-3273

$102 \mathrm{Lu} \mathrm{X}$, Dou H, Gao B, et al. A flexible graphene/multiwalled carbon nanotube film as a high performance electrode material for supercapacitors. Electrochim Acta, 2011, 56: 5115-5121

103 Pham DT, Lee TH, Luong DH, et al. Carbon nanotube-bridged graphene 3D building blocks for ultrafast compact supercapacitors. ACS Nano, 2015, 9: 2018-2027 
104 Du P, Hu X, Yi C, et al. Self-powered electronics by integration of flexible solid-state graphene-based supercapacitors with high performance perovskite hybrid solar cells. Adv Funct Mater, 2015, 25: 2420-2427

105 El-Kady MF, Ihns M, Li M, et al. Engineering three-dimensional hybrid supercapacitors and microsupercapacitors for high-performance integrated energy storage. Proc Natl Acad Sci USA, 2015, 112: 4233-4238

106 Xie B, Wang Y, Lai W, et al. Laser-processed graphene based micro-supercapacitors for ultrathin, rollable, compact and designable energy storage components. Nano Energ, 2016, 26: 276285

107 Xiong Z, Liao C, Han W, et al. Mechanically tough large-area hierarchical porous graphene films for high-performance flexible supercapacitor applications. Adv Mater, 2015, 27: 4469-4475

108 Xie J, Sun X, Zhang N, et al. Layer-by-layer $\beta$-Ni(OH $)_{2} /$ graphene nanohybrids for ultraflexible all-solid-state thin-film supercapacitors with high electrochemical performance. Nano Energ, 2013, 2: 65-74

109 Wu ZS, Tan YZ, Zheng S, et al. Bottom-up fabrication of sulfurdoped graphene films derived from sulfur-annulated nanographene for ultrahigh volumetric capacitance micro-supercapacitors. J Am Chem Soc, 2017, 139: 4506-4512

110 Ai W, Luo Z, Jiang J, et al. Nitrogen and sulfur codoped graphene: multifunctional electrode materials for high-performance Li-ion batteries and oxygen reduction reaction. Adv Mater, 2014, 26: 6186-6192

111 Wu ZS, Winter A, Chen L, et al. Three-dimensional nitrogen and boron co-doped graphene for high-performance all-solid-state supercapacitors. Adv Mater, 2012, 24: 5130-5135

112 Chen X, Chen X, Xu X, et al. Sulfur-doped porous reduced graphene oxide hollow nanosphere frameworks as metal-free electrocatalysts for oxygen reduction reaction and as supercapacitor electrode materials. Nanoscale, 2014, 6: 13740-13747

113 Dong XC, Xu H, Wang XW, et al. 3D graphene-cobalt oxide electrode for high-performance supercapacitor and enzymeless glucose detection. ACS Nano, 2012, 6: 3206-3213

114 Choi BG, Yang MH, Hong WH, et al. 3D macroporous graphene frameworks for supercapacitors with high energy and power densities. ACS Nano, 2012, 6: 4020-4028

$115 \mathrm{Xu} \mathrm{Y,} \mathrm{Lin} \mathrm{Z,} \mathrm{Huang} \mathrm{X,} \mathrm{et} \mathrm{al.} \mathrm{Flexible} \mathrm{solid-state} \mathrm{supercapacitors}$ based on three-dimensional graphene hydrogel films. ACS Nano, 2013, 7: 4042-4049

116 Shi JL, Du WC, Yin YX, et al. Hydrothermal reduction of threedimensional graphene oxide for binder-free flexible supercapacitors. J Mater Chem A, 2014, 2: 10830

117 Shao Y, El-Kady MF, Lin CW, et al. 3D freeze-casting of cellular graphene films for ultrahigh-power-density supercapacitors. Adv Mater, 2016, 28: 6719-6726

118 Deville S. Freeze-casting of porous ceramics: a review of current achievements and issues. Adv Eng Mater, 2008, 10: 155-169

119 Yu P, Zhao X, Huang Z, et al. Free-standing three-dimensional graphene and polyaniline nanowire arrays hybrid foams for highperformance flexible and lightweight supercapacitors. J Mater Chem A, 2014, 2: 14413-14420

120 Jurewicz K, Vix-Guterl C, Frackowiak E, et al. Capacitance properties of ordered porous carbon materials prepared by a templating procedure. J Phys Chem Solids, 2004, 65: 287-293

121 Álvarez S, Blanco-López MC, Miranda-Ordieres AJ, et al. Electrochemical capacitor performance of mesoporous carbons obtained by templating technique. Carbon, 2005, 43: 866-870

122 Li HQ, Luo JY, Zhou XF, et al. An ordered mesoporous carbon with short pore length and its electrochemical performances in supercapacitor applications. J Electrochem Soc, 2007, 154: A731

123 Zhi J, Zhao W, Liu X, et al. Highly conductive ordered mesoporous carbon based electrodes decorated by $3 \mathrm{D}$ graphene and 1D silver nanowire for flexible supercapacitor. Adv Funct Mater, 2014, 24: 2013-2019

124 Qin $\mathrm{T}$, Wan Z, Wang Z, et al. 3D flexible $\mathrm{O} / \mathrm{N}$ co-doped graphene foams for supercapacitor electrodes with high volumetric and areal capacitances. J Power Sources, 2016, 336: 455-464

Acknowledgements This work was supported by the National Natural Science Foundation of China (21503116), Taishan Scholars Program of Shandong Province (TSQN20161004) and the Youth 1000 Talent Program of China.

Author contributions $\mathrm{Li} \mathrm{K}$ and Zhang J conceived and wrote the paper. All authors discussed and commented on the manuscript.

Conflict of interest The authors declare that they have no conflict of interests. 


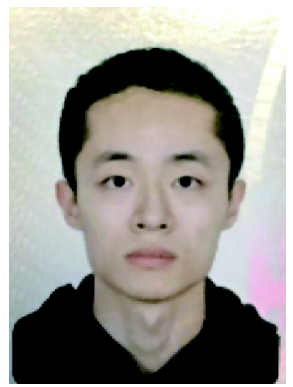

Kang Li obtained his bachelor degree from Nanjing University, and now is a graduate student in Shandong University under the supervision of Prof. Jintao Zhang. His research interest focuses on the carbon-based nanomaterials for flexible energy storage devices.

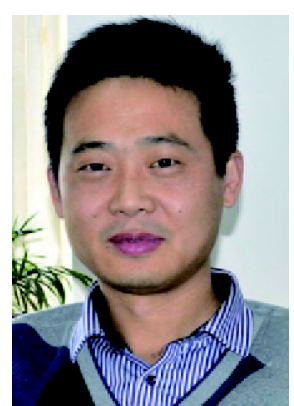

Jintao Zhang obtained his PhD degree from the National University of Singapore in 2012. Prior to joining Shandong University as a full professor, he has been a postdoctoral fellow at Nanyang Technological University and Case Western Reserve University. His research interests include the rational design \& synthesis of advanced materials for electrochemical energy storage and conversion devices (e.g., metal-air batteries, supercapacitors and fuel cells) and electrocatalysis.

\section{碳纳米管和石墨烯材料在柔性超级电容器中的应用}

李康, 张进涛 ${ }^{*}$

摘要 随着柔性电子器件的发展, 对与之相匹配的柔性储能器件的需求越来越大. 柔性超级电容器因其具有快速的充放电倍率、长循环 寿命和良好的柔韧性而受到广泛关注. 碳纳米管和石墨烯拥有良好的机械性能和多样的结构, 不同的微纳结构对电极的电化学性能和机 械性能有较大的影响. 本文总结了近期以碳纳米管和石墨烯为电极材料的柔性超级电容器研究进展, 重点概括了不同柔性电极的合成方 法和结构特点, 并分析了构建柔性超级电容器的容量性能和机械性能. 此外, 还讨论了柔性超级电容器发展现存的挑战和未来的前景. 